\title{
Understanding factors that influence unintentional insider threat: a framework to counteract unintentional risks
}

\author{
Neeshe Khan ${ }^{1}\left[\right.$ [ Robert J. Houghton ${ }^{1} \cdot$ Sarah Sharples ${ }^{1}$
}

Received: 19 January 2021 / Accepted: 2 October 2021 / Published online: 28 October 2021

(c) The Author(s) 2021

\begin{abstract}
The exploitation of so-called insiders is increasingly recognised as a common vector for cyberattacks. Emerging work in this area has considered the phenomenon from various perspectives including the technological, the psychological and the sociotechnical. We extend this work by specifically examining unintentional forms of insider threat and report the outcomes of a series of detailed Critical Decision Method (CDM) led interviews with those who have experienced various forms of unwitting cybersecurity breaches. We also articulate factors likely to contribute firmly in the context of everyday work-asdone. CDM's probing questions were used to elicit expert knowledge around how decision making occurred prior, during and post an unintentional cyber breach whilst participants were engaged in the delivery of cognitive tasks. Through the application of grounded theory to data, emerging results included themes of decision making, task factors, accidents and organisational factors. These results are utilised to inform an Epidemiological Triangle to represent the dynamic relationship between three vectors of exploit, user and the work environment that can in turn affect the resilience of cyber defences. We conclude by presenting a simple framework, which for the purposes of this work is a set of recommendations applicable in specific scenarios to reduce negative impact for understanding unintentional insider threats. We also suggest practical means to counteract such threats rooted in the lived experience of those who have fallen prey to them.
\end{abstract}

Keywords Critical Decision Method · Cybersecurity · Human factors · Insider threat $\cdot$ Sociotechnical framework · Sociotechnical systems

\section{Introduction}

The spread of internet-enabled services and devices into the workplace has led to significant gains in productivity and efficiency (Schuh et al. 2014). However, this technology also offers potential vulnerabilities for criminals, industrial saboteurs and extortionists to exploit, a matter of increasingly widespread concern and media interest (e.g., Dice 2020). Aside from what might be considered traditional hacking of digital systems at a technical level (e.g., Goethals and Hunt 2019), there is increasing prevalence of cyberattacks

Neeshe Khan

Neeshe.Khan@nottingham.ac.uk

Robert J. Houghton

Robert.Houghton@nottingham.ac.uk

Sarah Sharples

Sarah.Sharples@nottingham.ac.uk

1 University of Nottingham, Nottingham, UK that require the unwitting participation of innocent individuals in terms of opening an attachment, clicking on a rogue link or otherwise inadvertently completing the last action that compromises a system (Verizon 2020). This innocent facilitation of cybercrime by insiders is considered a subset of "Insider Threat (IsT)" known as unintentional/accidental insider threat with the remainder of the category known as intentional/malicious insider threat comprising of deliberate and malicious actions carried out by disaffected or mercenary employees within an organisation (Mundie et al. 2013). Previous work in this area has tended to merge these two forms of insider threat and falls broadly into three areas of investigation: the largely technical including the potential for AI or machine learning to actively block threats (e.g., Morel 2011); the psychological which has considered whether personality variables can be associated with increased risk of an individual committing a cyber-breach either deliberately or accidentally (e.g., Hunker and Probst 2011; Hadlington 2018) and a more organisational approach that has tended to emphasise governance of IT systems in organisations 
and management practices intended to increase security (e.g., Cappelli et al. 2008, CERT 2013). Often technical, psychological and organisational approaches are combined to inform sociotechnical defences. Some contact has also been made between the nascent cybercrime literature and the larger and more established literature on safety and accident prevention (e.g., the application of Reason's Generic Error Management System to the case of cyber-breaches, Liginlal et al. 2009) and attempts made to position insider threat within a sociotechnical framework (e.g., Nurse et al. 2014).

In the work presented in this paper we extend this sociotechnical perspective to examine unintentional insider threat specifically to investigate the individual and contextual factors that exist in the moment of an inadvertent cybersecurity breach: either sending email in a way that breaches data protection regulations or clicking on a malicious link in a received email. The approach taken was to conduct an in-depth exploration of decision factors at the time of the breach through an adaptation of the well-known Critical Decision Method (Klein et al. 1989). Whilst this method is normally associated with capturing expertise in decision making (e.g., with Fire Commanders, Pilots, Surgeons, Controllers, see Plant and Stanton 2013; Pauley et al. 2011; Bearman and Bremner 2013) our view of cybersecurity breaches here is that whilst viewed retrospectively as errors, they take place in the context of otherwise expert work that the method is useful in capturing the complexity of and indeed, in as far as cyberattacks rely on tricking people, may be considered the exploitation of expertise built around cyber breaches as experienced (rather than, perhaps, as imagined). Our aim in doing this is ultimately to offer a richer picture of why these events occur in order to design interventions to reduce their frequency or mitigate their impact. In order to position our approach, we begin by briefly reviewing extant literature on inadvertent insider threat in cybersecurity.

\section{Background}

Generally, IsT can be understood as the vulnerability in systems and infrastructure pertaining to assets through the action or inaction of individuals or 'insiders'. This vulnerability arises from individual's access privileges and proximity to and knowledge of systems and a threat's severity can be affected by insider's skillsets and motivations. For the purposes of this work IsT and insiders are defined as follows:

Actions (encompassing skills, rules and knowledgebased behaviour) or inactions of individuals or groups wittingly or unwittingly that cause loss or harm to the security of an individual, organization or the larger society, without differentiating between cyber or physical parameters. The individuals have authorized access (physical and/or cyber) to physical assets and to confidential information in order to perform a function for themselves or an organization, which results in compromised safety or a cybersecurity breach.

Based on insiders' intentions two types of IsT exist: intentional (also known as malicious) and unintentional (also known as accidental) which can be posed by an individual or a group (Predd et al. 2008) and it is unintentional IsT (UIsT) that is of interest to this work. Unintentional insiders do not mean to harm the organisation, but their actions or inactions can put assets and operations of the organisation at risk, affecting systems' confidentiality, integrity and availability (CIA security triad) and resulting in a cyber incident or breach. Examples of this are evidenced in real life through daily reports of successful ransomware attacks experienced by organisations where well-intentioned employees unwittingly interact with malicious content or accidentally exposed personal data.

\subsection{Technical defences}

Technical defences involve software or algorithmic solutions that safeguard against cyber threats including IsT. Goethals and Hunt (2019) divide all cyberspace operations as offensive or defensive. Stemming from conventional security thought, Defensive Cyberspace Operations (DCOs) aim to defend against attacks. DCOs are further categorised as either passive or active cyber defences against threats. Goethals and Hunt (2019) list passive cyber defences as adopting best practice in setting up systems, system monitoring and exchanging information. Thus, passive defences would include measures such as configuration management, encryption (symmetric and asymmetric), configuration monitoring, data management (storage, access and architecture) and software updates. Passive defences can also include defences encompassed within Intrusion Prevention/Detection Systems such as anomaly based, signature based and stateful protocol detection (Magklaras and Furnell 2002). Active cyber defences involve actively defending against threats through intelligent interference but must be legally permissible in the country of operation. Active defensive techniques can include computer forensics (network and system based, mobile devices and email), Intrusion Prevention Systems (network based, wireless, network behaviour analysis and host based), cyber-physical systems, stateful protocol detection and anomaly-based identification (Zargar et al. 2016) and deception software (honeypots, decoys and address hopping). Both passive and active defences are used as solutions to guard against IsT through monitoring systems' behaviour and containing the spread of malicious software in networks in the event of an attack. Popular research now includes developing machine learning algorithms and the use of AI for smart cybersecurity. Algorithm 
based solutions are popular and conventional in computer science and while these techniques can strengthen baseline defences such as those adopted in passive defences, active defences are not effective for guarding against UIsT alone due to its unintentional nature.

With concerns over technical defences being singularly focused on algorithmic elements numerous technical solutions have emerged that focus on human and process elements to identify insiders who might pose a threat (Ani et al. 2018). This is largely accomplished through considering individual psychological and behavioural characteristics. Such technical solutions can include detection of anomalies based on individual and group network behaviour (Legg et al. 2017; Agrafiotis et al. 2015; Chattopadhyay et al. 2018), background checks and rule breaking behaviour (Bishop et al. 2008; Greitzer and Hohimer 2011; Kammueller and Probst 2013; Ogiela and Ogiela 2012). While such solutions highlight the need for effective defences there are notable limitations that might arise from implementation. For example, the lack of professionally trained resources to assess psychological traits, targeting of individuals, creating a conflict environment at work and considering psychological aspects in isolation from other factors that exist in complex environments.

\subsection{Sociotechnical defences}

Sociotechnical defences include technical, individual (including psychological traits) and organisational factors that influence IsT to design solutions for complex environments. Whilst there are numerous sociotechnical solutions being proposed we will discuss works that are the most relevant to safeguarding against unintentional IsT.

The National Cyber Security Centre (NCSC 2012) '10 Steps to Cybersecurity' guide proposes a mixture of technical, individual and organisational defences that can in-turn guard against IsT. This guide includes PCDs such as network security, malware prevention software, secure configuration and managing user privileges. Individual factors include monitoring user activity and devices. Organisational factors include educational and awareness training for users based on security policies. It is recommended that trainings be regularly audited for effectiveness and staff should be encouraged to attain formal qualifications to build in-house security skills. Organisational factors also include communicating risks and acceptable use of company systems, regulating the use of removable media devices and an incident management plan that is strengthened through specialised training and rehearsed through drills. Organisations are also advised to promote an incident reporting culture that empowers staff to share poor practices without the fear of being blamed. While these suggestions are effective the guide also contradicts itself and states individuals should be held personally responsible for deviations in security policy as well as formal disciplinaries and actionable penalties that are enforced for IsT, which is effective for intentional IsT but gravely damaging for UIsT. Reprimands or penalties for UIsT can easily result in feelings of injustice, bitterness, embarrassment, blame, low morale and demotivation in an organisation.

One of the most popular and recognised frameworks is the MERIT insider threat model developed by CERT Program at Carnegie Mellon University's Software Engineering Institute (Keeney et al. 2005; Cappelli et al. 2007, 2008). Their work involved evaluating case studies based on real world scenarios in interactive learning environments to mimic complex systems involving humans and technology. MERIT model is focused on evaluating insiders' skills, motivations and opportunities to become an IsT. Determining the motivations of insiders includes individual psychological and behavioural profiling (including background checks). The framework also considers organisational characteristics and recommendations include awareness training, creation, implementation and maintenance of security policy, organisational interventions for insiders exhibiting concerning behaviour and managing employee expectations and various PCDs to secure systems and access points. CERT (2013) links UIsT to limitations in human performance and fallibility. Human errors would thus increase in likelihood with time pressures to deliver tasks, lack of knowledge, difficulty of the task and load on cognitive factors such as inattention. Limitation of this model are that individuals might not report concerning behaviour due to multiple factors that are at play in work contexts (Bell et al. 2019). It is also problematic to propose simplified solutions for a complex problem such as UIsT and viewing the system as decomposable to propose solutions in isolation from other parts (Hollnagel et al. 2015).

The combination of technical defences alongside psychological traits to identify UIsT has enjoyed growing popularity over the last decade with a range of psychological measures being adopted to detect UIsT (Hadlington 2018). A framework developed by Nurse et al. (2014) furthers CERT's work discussed above. Similar to CERT, UIsT is associated to factors such as time pressures but these are positioned to stem from task objectives. Evaluating accidents in this format can provide insights into the root causes and allow corrective measures to be implemented. While this framework provides a better understanding of why errors occur, it has limited features to consider (not applicable categories) for UIsT and subsequently lacks concrete recommendations to tackle UIsT.

Liginlal et al. (2009) produced a framework for privacy breaches based on GEMs that includes an 'error management program' through examining errors. This program proposes looking at the root causes for errors, creating a defence-in-depth strategy that works to avoid, intercept and correct errors and periodically evaluating processes. It states that error avoidance emerges from poor feedback and lack of 
experience which can be overcome through training. Errors can be intercepted through better system design such as displays, monitoring and alarms. Errors can be corrected through conducting a timely investigation, an in-depth analysis of why the error occurred without blaming individuals and proposing solutions. Recommendations to strengthen organisational factors includes: careful consideration before implementing new systems as it can lead to new errors, developing explicit and effective processes to conduct tasks supported through training, individual's resistance to change which can result in lower errors being reported which is strengthened through focusing on situations and systems rather than individuals and lastly monitoring work-related fatigue.

The last framework that is relevant to our work is developed by Greitzer et al. (2018) called 'Sociotechnical and Organizational Factors for Insider Threat' or SOFIT. SOFIT incorporates a combination of PCDs and ACDs, 271 individual behavioural and psychological factors (such as dark triad, dynamic states and personality dimensions) and 49 organisational factors to identify IsT (such as poor communication, inadequate training, ambiguous goals, stress, workload, blame culture, poor team management, poor system designs, environmental stressors, unrealistic deadlines, mismatch between expectations and abilities and morale). Limitations of this framework are the non-disclosure of the complete list of factors that are considered within each of the three categories, relying on individuals to report observed behaviours and non-disclosure of algorithm used as a black box to make assessments of potential risks.

The relevant technical and sociotechnical solutions discussed above propose defences from a standpoint of humans are the weakest link. When such a standpoint is adopted it is this human link that must be isolated, targeted and forced to conform should anomalies occur. Arguably technical defences promote solutions through a reductionist approach based on individual psychological or behavioural profiling. On the other hand, sociotechnical solutions consider a larger set of components in complex systems such as organisational culture and workload. However, they largely rely on reporting culture and an institutional entity that can surveillance procedural adherence and reprimand deviation instead of adopting a Joint Cognitive Systems approach (Woods and Hollnagel 2006). This policing on micro levels can be extremely challenging and simultaneously diminishes the importance of recognising factors that might come into play when complex tasks requiring cognitive resources are delivered in real world settings. In fact, research from a human factors' perspective is only just starting to emerge that considers human errors in cognitive tasks (Canham et al. 2020) or organisational factors (Greitzer et al. 2018) to address UIsT or the loss of attention to some areas in dynamic events (Vanderhaegen et al. 2020).

Furthermore, the proposed solutions aim to address intentional and unintentional insider threats simultaneously with a singular focus that results in a 'one size fits all' solution. With the bundling of unintentional and malicious threats together the solutions result in being too severe for unintentional errors, self-contradicting or having limited applicability to UIsT, which is a limitation recognised by some of the authors themselves. In order to devise effective solutions that work UIsT must be examined separately to malicious/ intentional IsT and afford individual ways of working within complex systems to enable human brilliance. We thus conducted a study to investigate how decision making occurs in complex cognitive tasks that resulted in UIsT being realised to identify factors that influence UIsT.

\section{Methods}

To encourage a rich discussion around naturalistic decision making (NDM) when engaging with activities that resulted in cyber breaches, we used Critical Decision Method for Eliciting Knowledge (CDM) (Klein et al. 1989). CDM was particularly fitting for the design of our interview questions as it focuses on a major event retrospectively with probing follow-up questions to guide discussions. These probing questions assist in eliciting expert knowledge about how decision making occurs in cognitive tasks. While we recognise that individuals are not experts at falling for cyber attacks, the interest is that breaches happen in the context of expert behaviour at work or in our personal lives. CDM is also widely used across various domains to help analyse decisions (Hoffman et al. 1998) and inform system development and design. While CDM is normally used in homogenous samples (different individuals performing the same task in the same environment) we used this method differently as all our participants performed various jobs and worked on assorted levels in organisations for different periods of time with their employers. However, they all made critical decisions in complex work or personal contexts that led to all of them experiencing a major event of a cyber breach.

CDM begins with a general question about the incident and in this study it was the cyber breach to construct an initial picture of the incident from the participant. CDM then provides probing questions based on the information shared by the participant. The use of CDM to explore cyber breaches was valuable as it allowed participant and the researcher to journey into an introspective in-depth examination of the incident. It also allowed conversation to flow naturally and provide overall consistency across discussions. Inclusion criteria consisted of participants having experienced one of the three scenarios in their personal or professional lives: (1) They had accidentally sent sensitive information to the wrong recipient; (2) They had accidentally clicked a link that resulted in Phishing, Ransomware or gave someone access to their private information; (3) They 
had clicked a link by mistake that gave someone access to their email account, social media account, bank account or personal device such as a laptop or mobile phone.

Structured approach and emergent themes approach are usually adopted for data analysis to compliment CDM (Wong 2004). Structured approach assumes a pre-existing framework within which data is then coded and the emergent themes approach focuses on the relationships between concepts. Presumptions about data was a particularly significant factor in our selection of methods as we did not want to make any assumptions prior to analysis. We consequently applied a grounded theory (GT) approach. Originating from sociology (Glaser and Strauss 1967) GT has since become widely adopted method by researchers (Muller and Kogan 2010). Key points in the data were identified and assigned codes, known as open coding. Codes where then compared against each other in the same interview and across interview transcripts, known as constant comparison method (Hoda et al. 2010). This was done until data reached saturation before commencing analysis.

\subsection{Participants}

Following ethical approval ten participants were recruited, eight from East Midlands and Greater London areas and two based internationally. Participants had to be over 18 years of age, have access to the internet and experienced one of the three scenarios in their personal or professional lives: (1) They had accidentally sent sensitive information to the wrong recipient; (2) They had accidentally clicked a link that resulted in Phishing, Ransomware or gave someone access to their private information; (3) They had clicked a link by mistake that gave someone access to their email account, social media account, bank account or personal device such as a laptop or mobile phone. Participants' scenario of breach, settings (personal or professional) and their occupation at the time the breach are shown in Table 1. Participants had a varying degree of experience ranging from mid-level to advanced, shown in Table 6.

Participants were not offered any compensation for sharing their experiences as part of this study and were provided associated materials describing the motives of the study prior to recruitment. As a result of snowball sampling some of the participants were known to the researcher (NK) in a professional context. Consent forms were completed and participants were given the opportunity to ask any questions prior to commencing any discussions.

\subsection{Data collection}

Data was collected between March to April 2020. Discussions were held individually with participants and audio and video recorded, generating approximately $9.5 \mathrm{hrs}$ of dialogue. Due to the Covid-19 outbreak in the UK, discussions were rescheduled to be held online on a platform most familiar to participants (Skype or Microsoft Teams) at a convenient date and time for them.

The full set of critical decision method based questions used in interviews are listed in Table 2. The researcher (NK) carried out discussions with participants and transcribed them verbatim from the digital recordings. NK also analysed and interpreted the data.

\subsection{Analysis}

Transcripts of the digital recordings were produced, anonymised and uploaded to QSR-NVivo software for coding. Transcripts were highlighted with colour ink and descriptively labelled based on open coding and constant comparison method. Once labelling was exhausted to the point no new labels could be generated, a three-stage analysis was conducted shown in Fig. 1. In stage 1, labels were organised thematically as 'Decision Making', 'Task Factors', 'Accidents' or 'Organisational Factors'. This thematic

Table 1 Participant scenarios, settings and associated professional fields at the time of cyber breach

\begin{tabular}{llll}
\hline Participant & Scenario & $\begin{array}{l}\text { Personal/profes- } \\
\text { sional setting }\end{array}$ & Field \\
\hline P1 & Accidently engaged with content that resulted in being hacked & Professional & Higher education (researcher) \\
P2 & Accidently sent sensitive information to the wrong recipient & Professional & Healthcare (grants manager) \\
P3 & Accidently sent sensitive information to the wrong recipient & Professional & Charity (grants manager) \\
P4 & Accidently engaged with content that resulted in being hacked & Professional & Higher education (researcher) \\
P5 & Accidently sent sensitive information to the wrong recipient & Professional & Charity (grants manager) \\
P6 & Accidently sent sensitive information to the wrong recipient & Professional & Charity (international partnerships) \\
P7 & Accidently engaged with content that resulted in being hacked & Personal & NA (student) \\
P8 & Accidently sent sensitive information to the wrong recipient & Professional & Think Tank (internee) \\
P9 & Accidently sent sensitive information to the wrong recipient & Professional & Food Retail (lawyer) \\
P10 & Accidently engaged with content that resulted in being hacked & Professional & Charity (accountant) \\
\hline
\end{tabular}


Table 2 Critical decision method based interview questions

\begin{tabular}{|c|c|}
\hline Decision (initial question) & $\begin{array}{l}\text { (a) Can you describe how you know if something is genuinely from the sender or not? } \\
\text { (b) How do you think this differs from someone with less experience with technologies? }\end{array}$ \\
\hline Knowledge (probe) & $\begin{array}{l}\text { Where do you think you acquired this knowledge to differentiate between content that is genuinely from the sender } \\
\text { and malicious content? }\end{array}$ \\
\hline Experience (probe) & $\begin{array}{l}\text { Thinking back to a specific time when you were cyberattacked, could you describe the incident from the time right } \\
\text { before you received the malicious content/virus to the time after you had/were about to click the link? }\end{array}$ \\
\hline Experience (probe) & Could you explain the sequence of events as they happened including how long each stage was? \\
\hline Cues (probe) & What were you seeing, reading or hearing that suggested that this content was genuine? \\
\hline Analogues (probe) & Were you reminded of any previous experience? \\
\hline Goals (probe) & If things went according to plan, what were you trying to achieve during the time the incident happened? \\
\hline Options and Basis (probe) & $\begin{array}{l}\text { (a) Did you consider any other actions to take prior to clicking the malicious content? } \\
\text { (b) [if applicable] How was this option selected and others rejected? }\end{array}$ \\
\hline Aiding (probe) & What training, knowledge or experience could have helped to avoid clicking this malicious content? \\
\hline Time pressure (probe) & $\begin{array}{l}\text { On a scale of } 1-5(1=\text { no pressure; } 5=\text { max pressure }) \text {, how much time pressure was involved in making this deci- } \\
\text { sion? }\end{array}$ \\
\hline Externals (probe) & $\begin{array}{l}\text { Do you think other/personal goals impacted how you made decisions when interacting with what might seem to be } \\
\text { malicious content? }\end{array}$ \\
\hline
\end{tabular}

Fig. 1 The three stages of data analysis

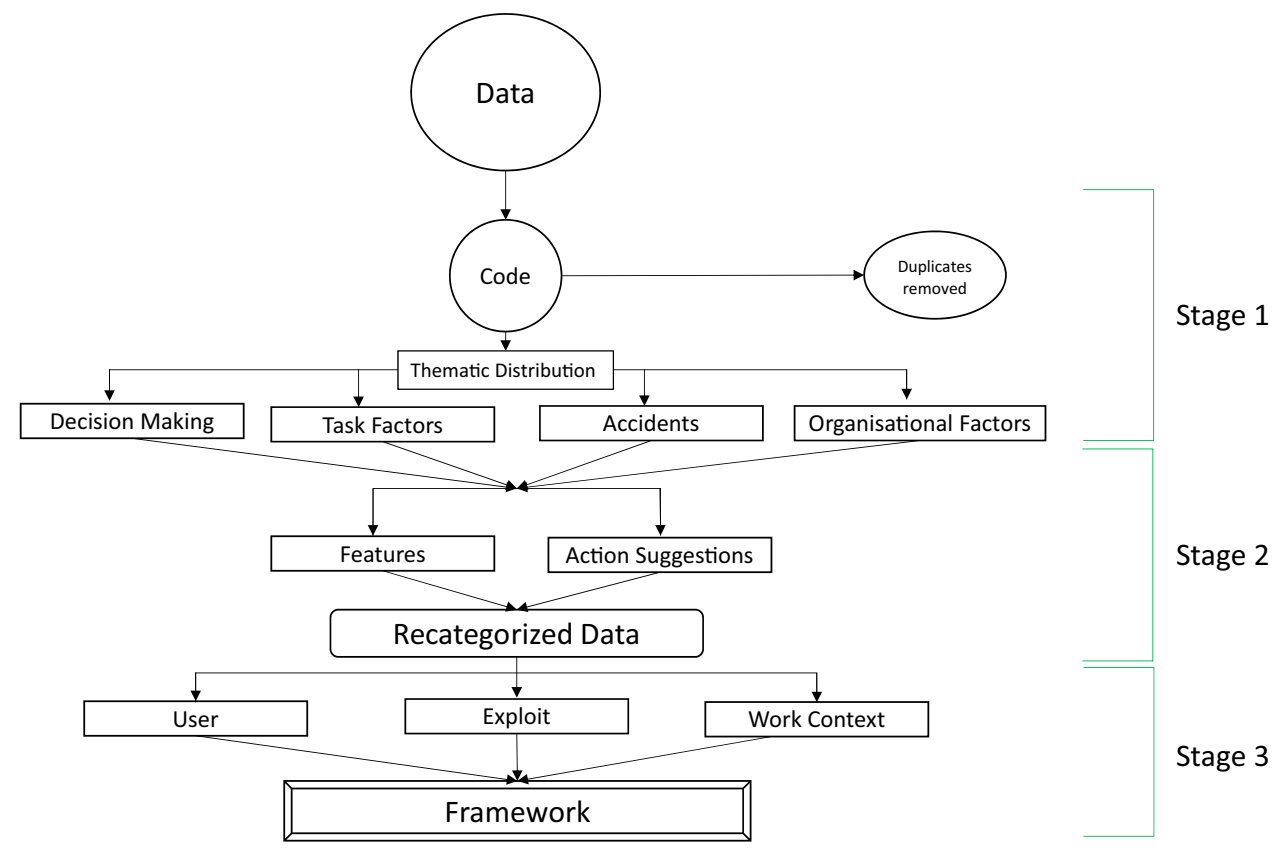

categorisation produced results that offered a better understanding of factors influencing UIsT. Stage 2 involved reorganizing codes as either features or actions. The results from this were used in stage 3 to further categorized according to the Epidemiological Triangle fields of 'user', 'exploit' or 'work context'. From this recategorized data a framework was developed to list 'inputs' and 'outputs' that can be used by organisations to identify, intervene and mitigate against UIsT. For the purposes of this work a framework is a set of recommendations applicable in specific scenarios to reduce negative impact.

Data was analysed with a critical realist view (Bhaskar 1989) where the researcher believes that participants can offer insights to cyber breaches through their experiences and that researchers also play a role in constructing that knowledge. An overarching epistemological position of relativism (Siegel 2004) was adopted which believes that all knowledge generated is context specific to individuals, society, culture and time.

\section{Results}

Four themes were identified in the analysis as factors that influence UIsT, presented in the following sections as: 'Decision Making', 'Task Factors', 'Accidents' and 'Organisational Factors', shown in Fig. 2. Numerous codes emerged 
Fig. 2 Thematic distribution of data

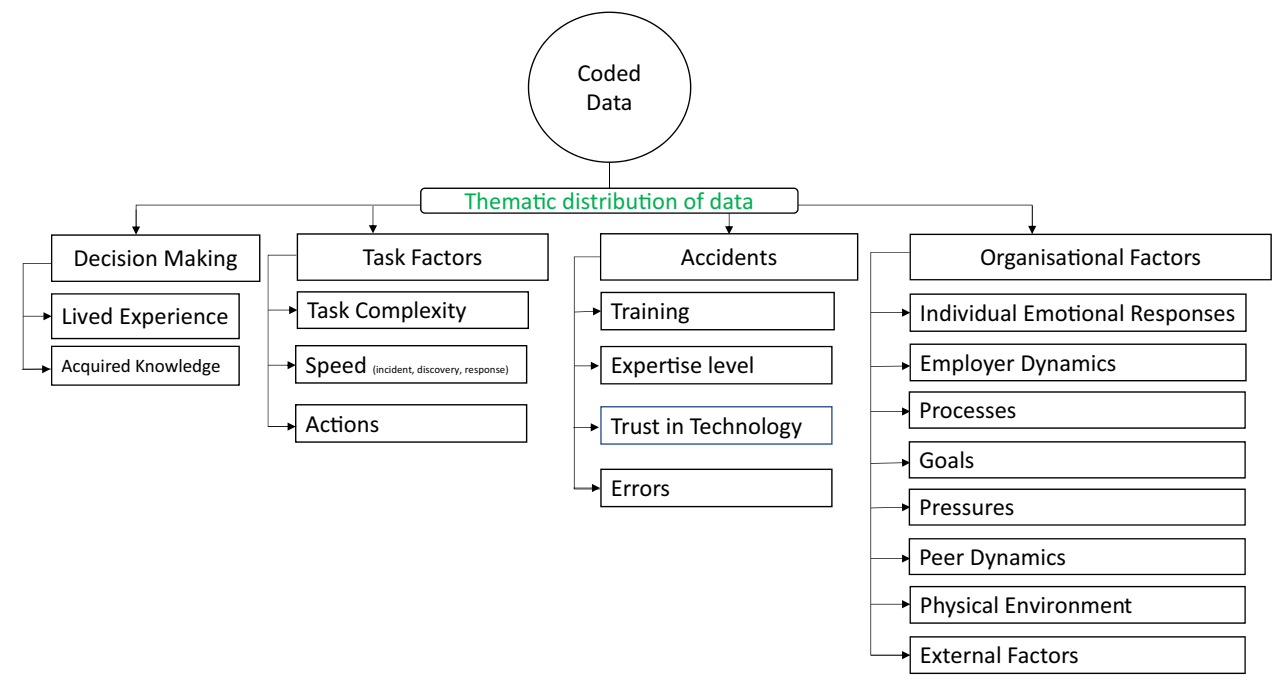

Table 3 Total code frequency for each theme

\begin{tabular}{lll}
\hline Theme name & Code frequency & $\%$ \\
\hline Decision making & 340 & 25 \\
Task factors & 238 & 17.5 \\
Accidents & 238 & 17.5 \\
Organisational factors & 530 & 40 \\
Total theme frequencies & 1346 & 100 \\
\hline
\end{tabular}

from the data and code frequencies listed in Tables 3 and 4 allowed us to cross check the weightings of our findings.

Quotes from participants are identified as; participant 1 (P1), participant 2 (P2) and so on. All participants are referred to as 'she' and 'her' regardless of their gender identification to maintain anonymity. Various quotes from participants and their respective context which support the results are evidenced in Table 5.

\subsection{Decision making (DM)}

All participants were asked to reflect on how their lived experience and acquired knowledge affected their DM when interacting with technologies. This was interesting as it gave an insight on how individuals might make informed decisions when identifying between malicious and nonmalicious content that included utilising cues and applying knowledge-based behaviour. Cues incorporated surface features (logo disparities or brand colours) and contextual features (typically around pre-existing expectations around who respondents would expect to hear from and the nature of their likely requests) which we discuss below. This theme was divided into two sub-categories: lived experience and acquired knowledge. The 'lived experience' category comprises of direct personal experiences which might contribute to 'acquired knowledge'. Acquired knowledge encompasses
Table 4 Code frequency for subcategories within each theme

\begin{tabular}{lll}
\hline Parent category & Subcategories & Frequency \\
\hline Decision making & Lived experience & 188 \\
Decision making & Acquired knowledge & 152 \\
Organisational factors & Individual & 111 \\
Organisational factors & External Factors & 96 \\
Organisational factors & Pressures & 91 \\
Organisational factors & Employer & 88 \\
Task factors & Speed (incident, discovery, & 83 \\
& response) & \\
Task factors & Actions & 80 \\
Organisational factors & Processes & 79 \\
Task factors & Complexity of task & 75 \\
Accidents & Training & 73 \\
Accidents & Expertise level & 61 \\
Accidents & Trust in Tech & 56 \\
Accidents & Errors & 48 \\
Organisational factors & Peer Dynamics & 31 \\
Organisational factors & Physical environment & 20 \\
Organisational factors & Goals & 14 \\
\hline
\end{tabular}

all channels used to build knowledge which can include lived experiences as well as other channels such formal classroom teaching, word-of-mouth or awareness campaigns.

\subsubsection{Lived experience}

All our participants shared a sense of reliance on their lived experiences which formed tacit knowledge to help them differentiate between genuine and malicious content, each with their own set of techniques and strategies to serve as defences. Techniques included clues from technical elements of the interaction such as the legitimacy of embedded web links, sender domain, font and logos. Participants also 


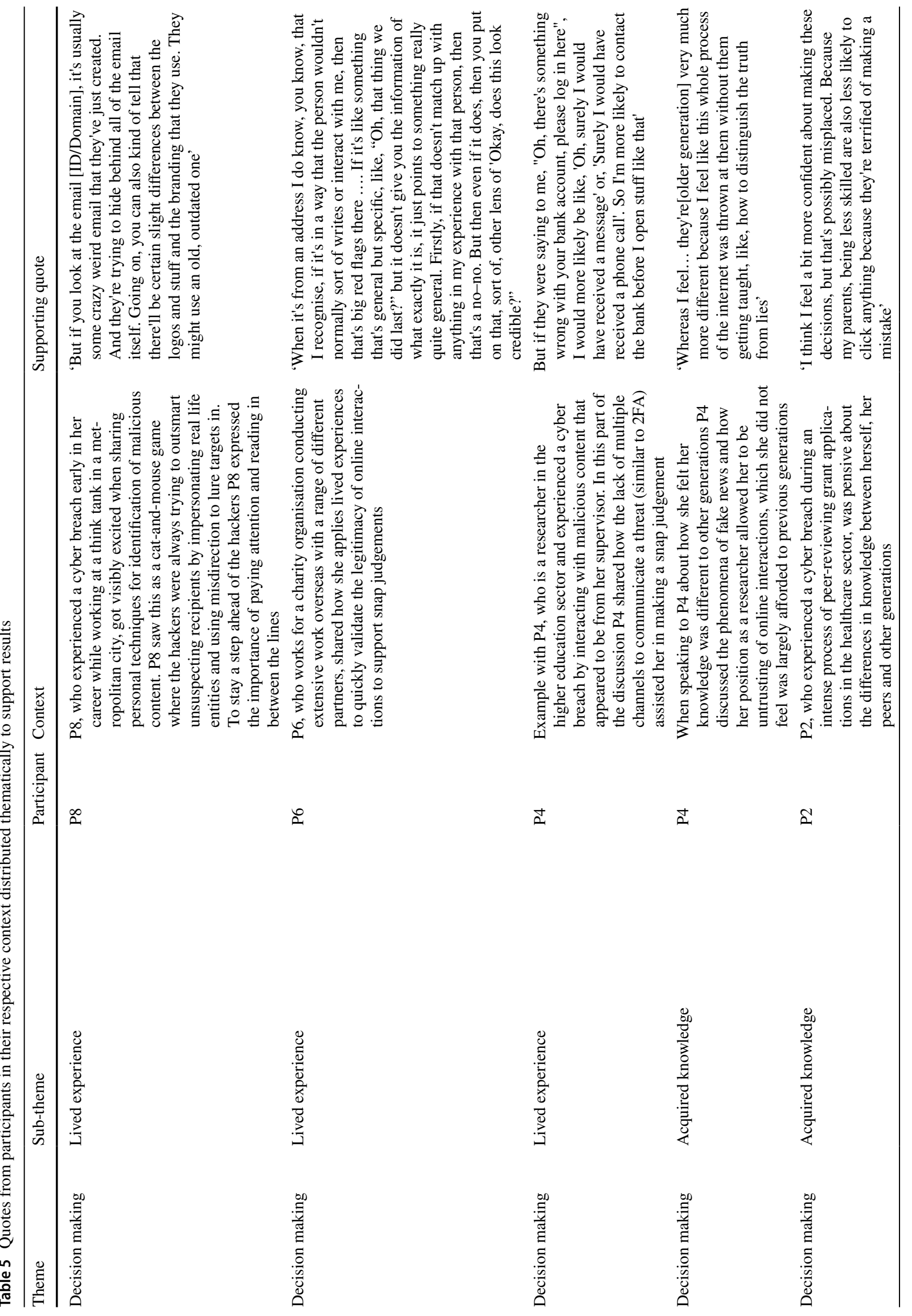




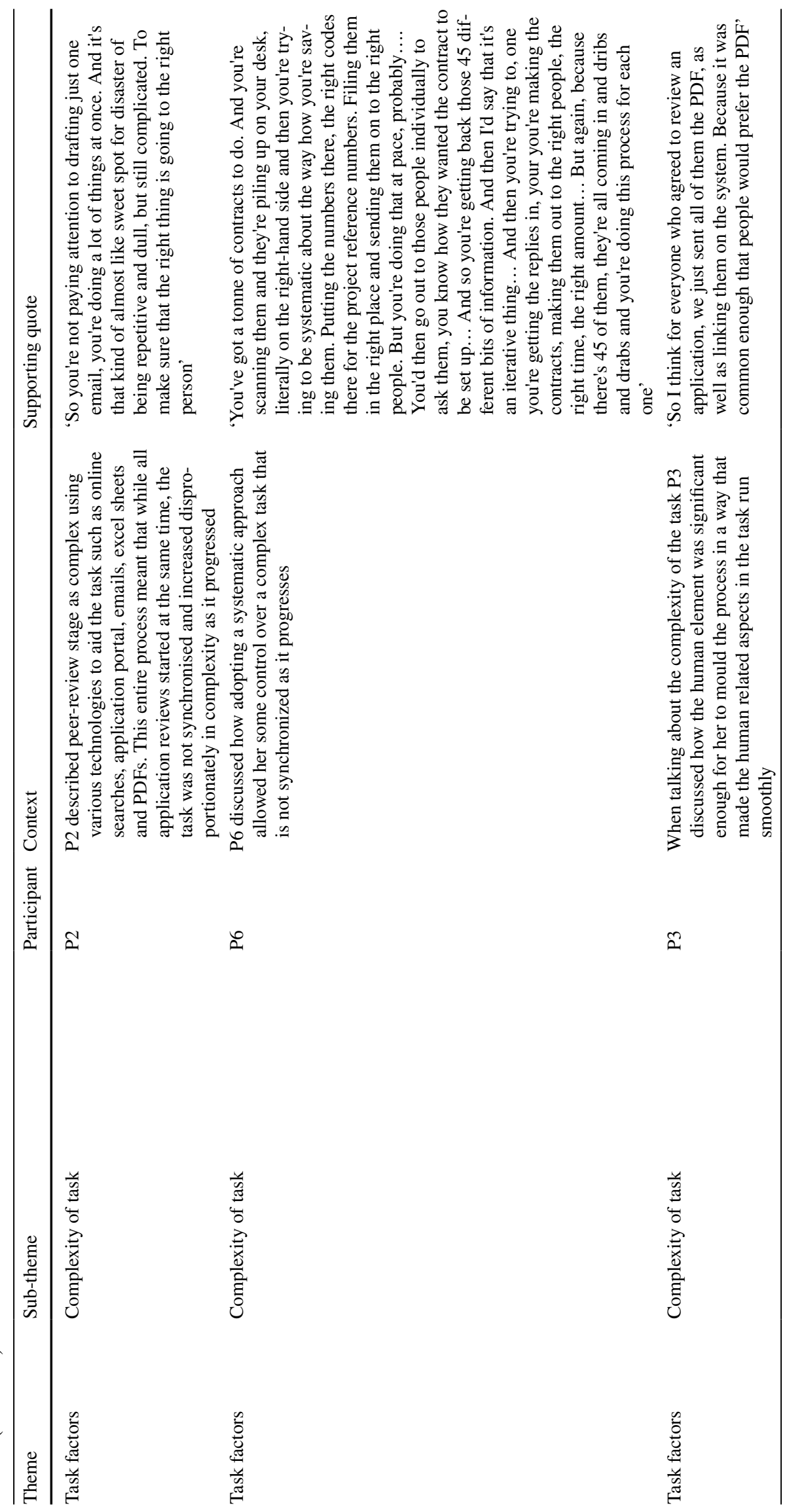




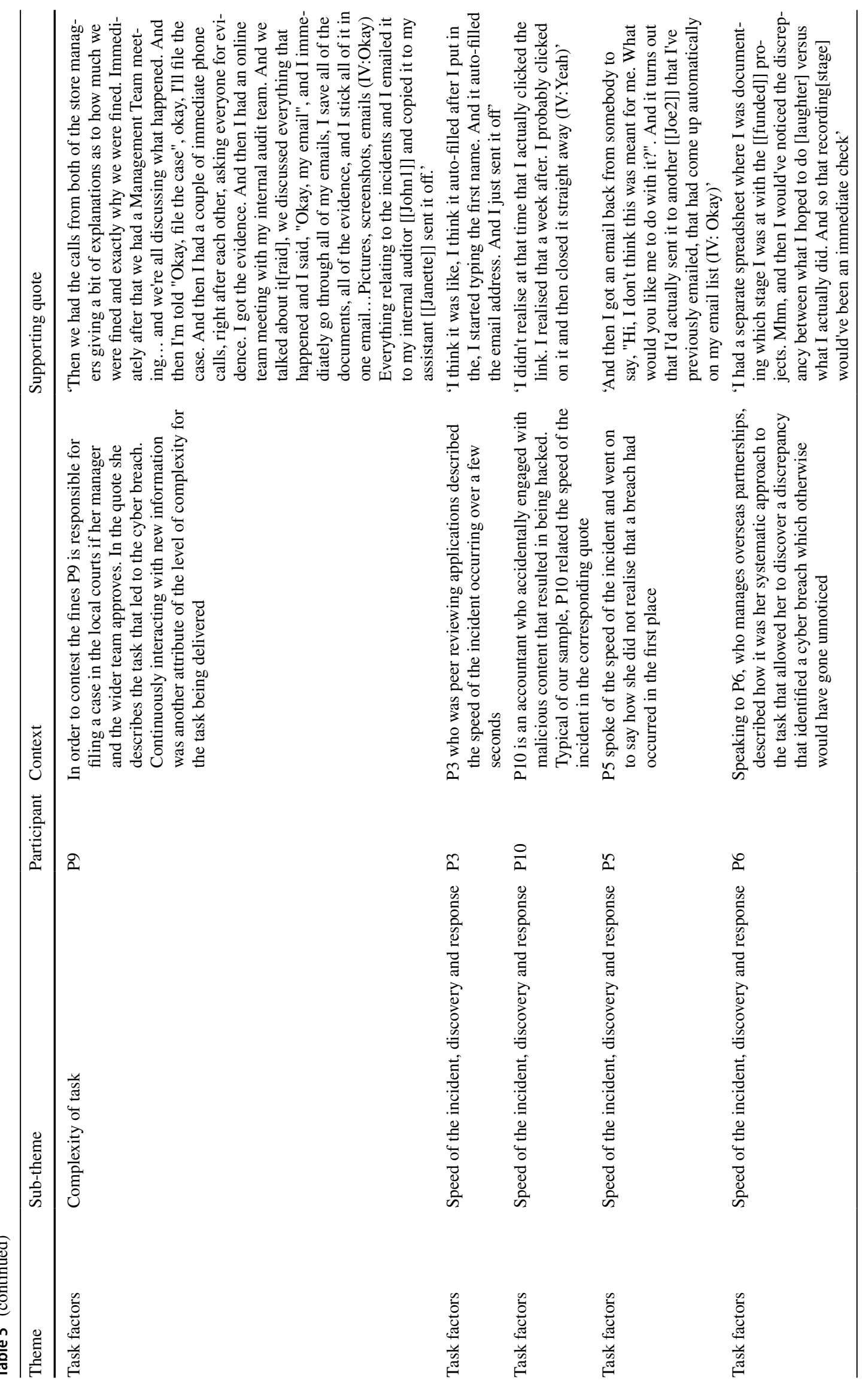




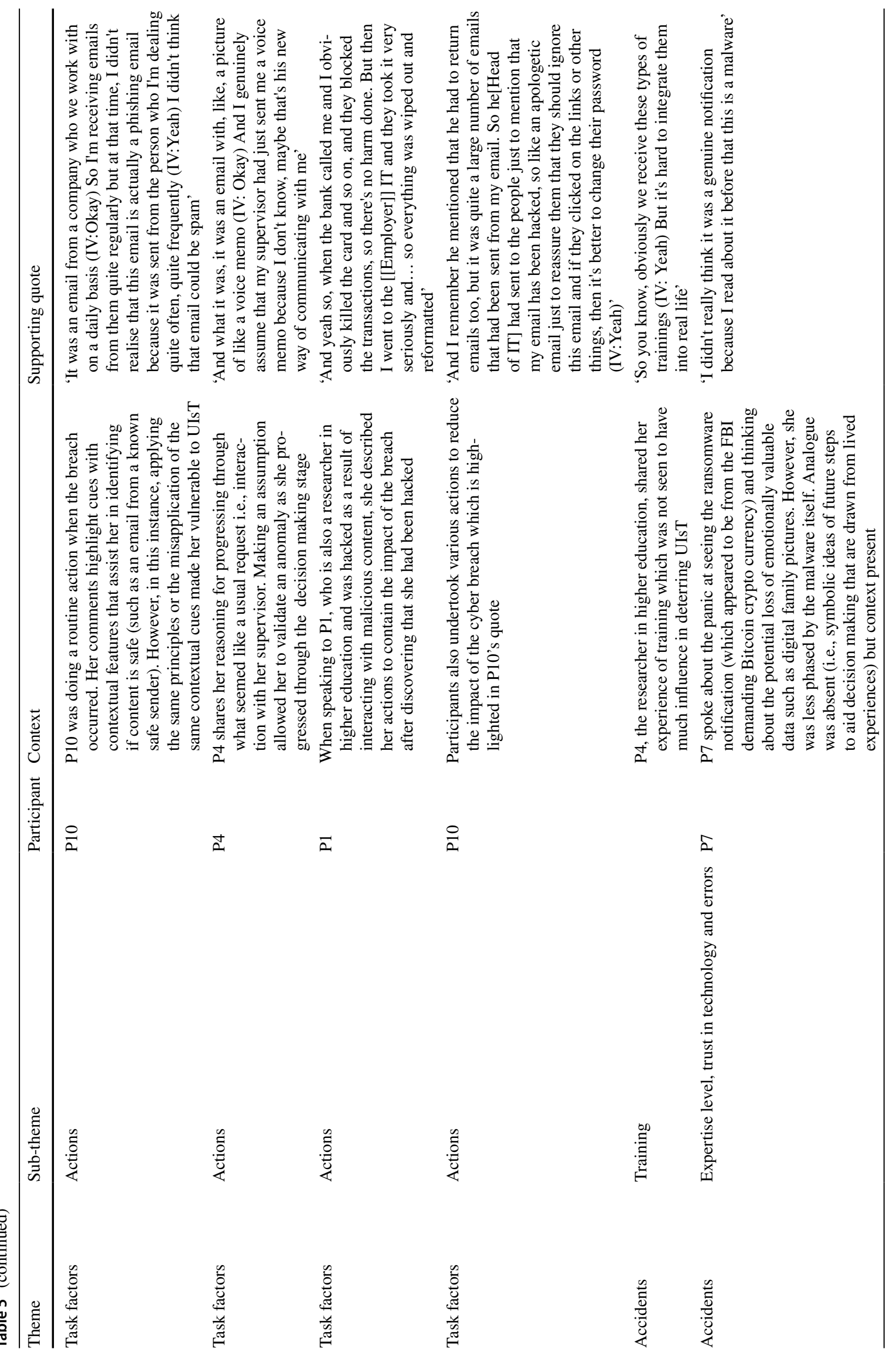




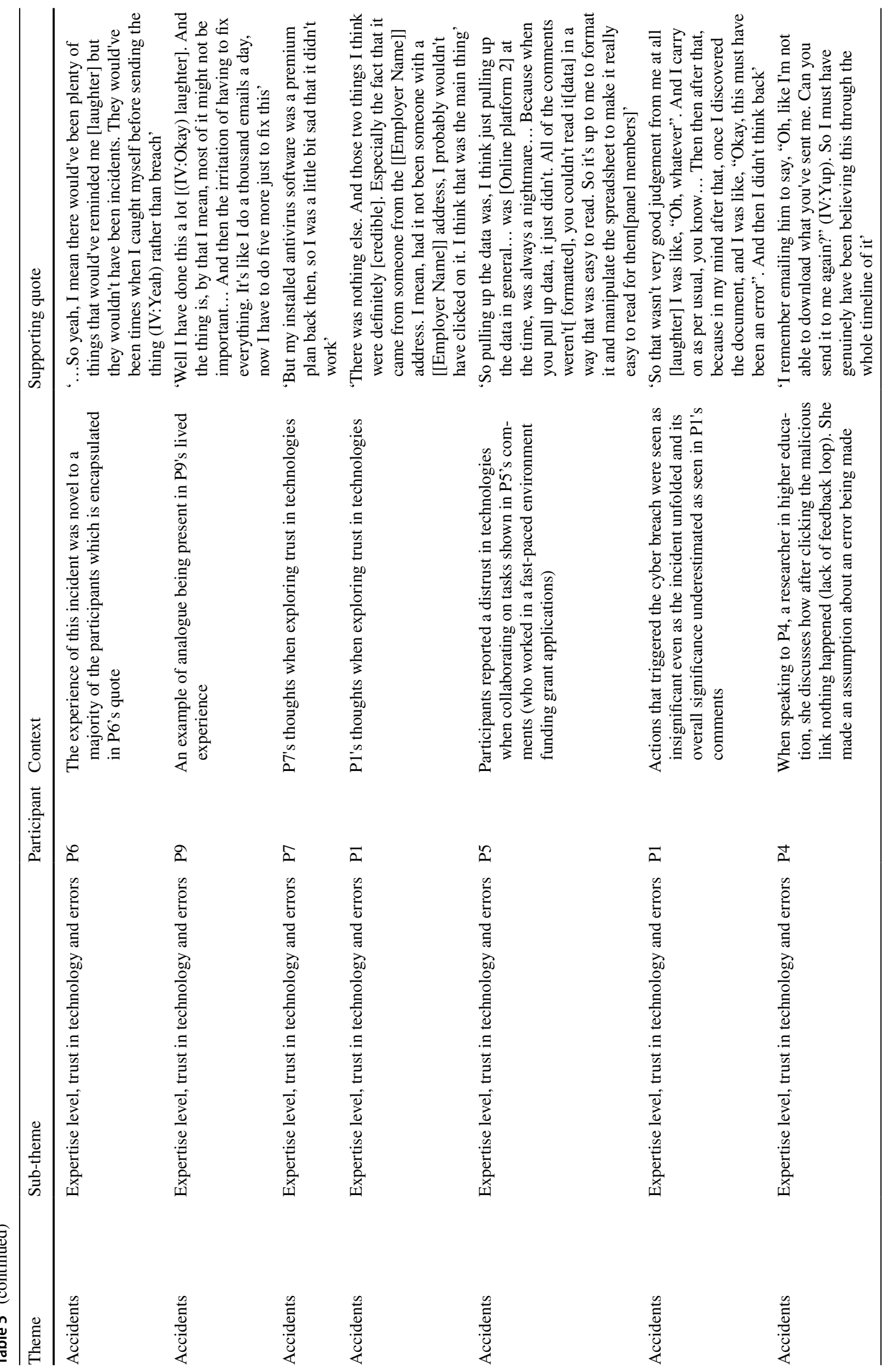




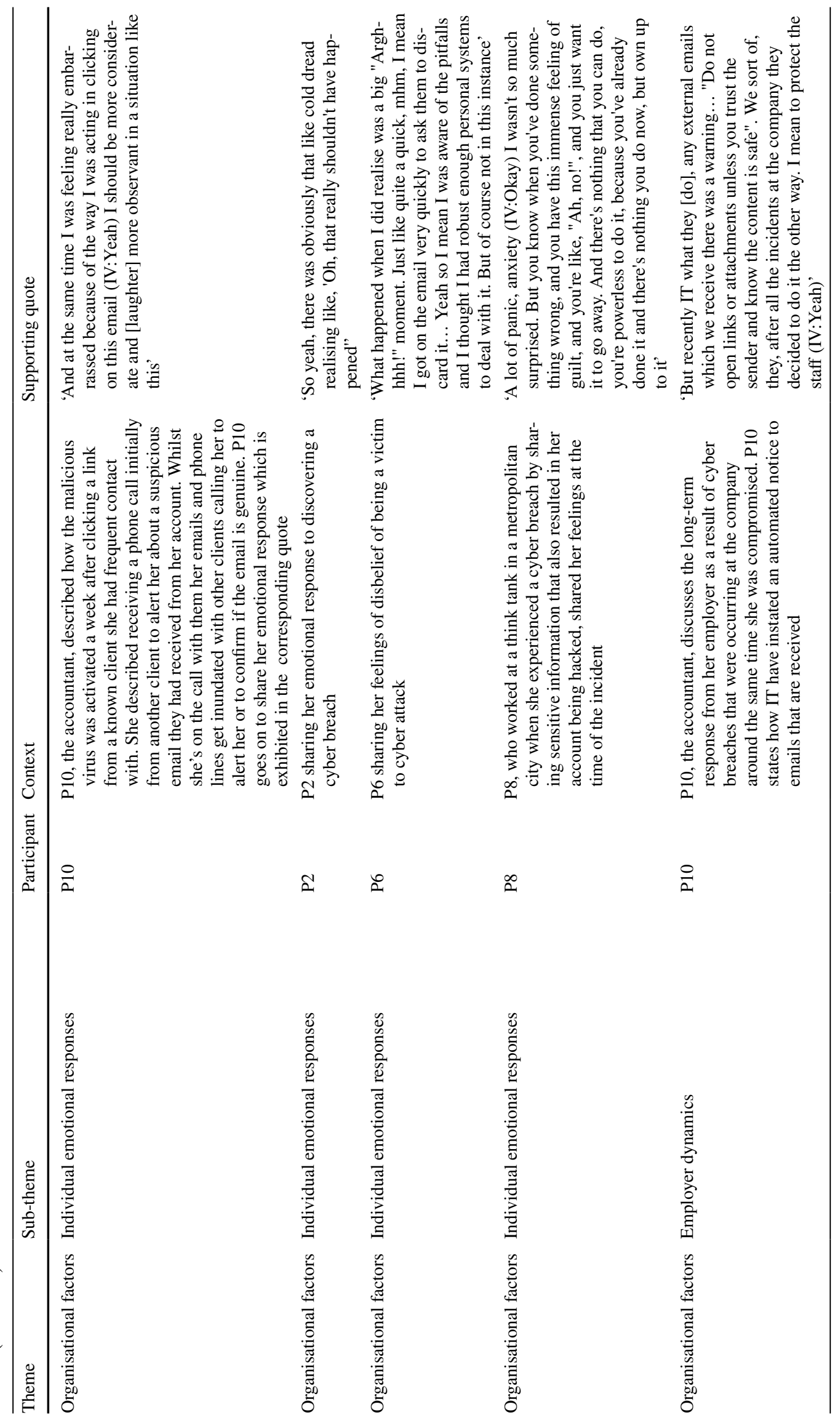




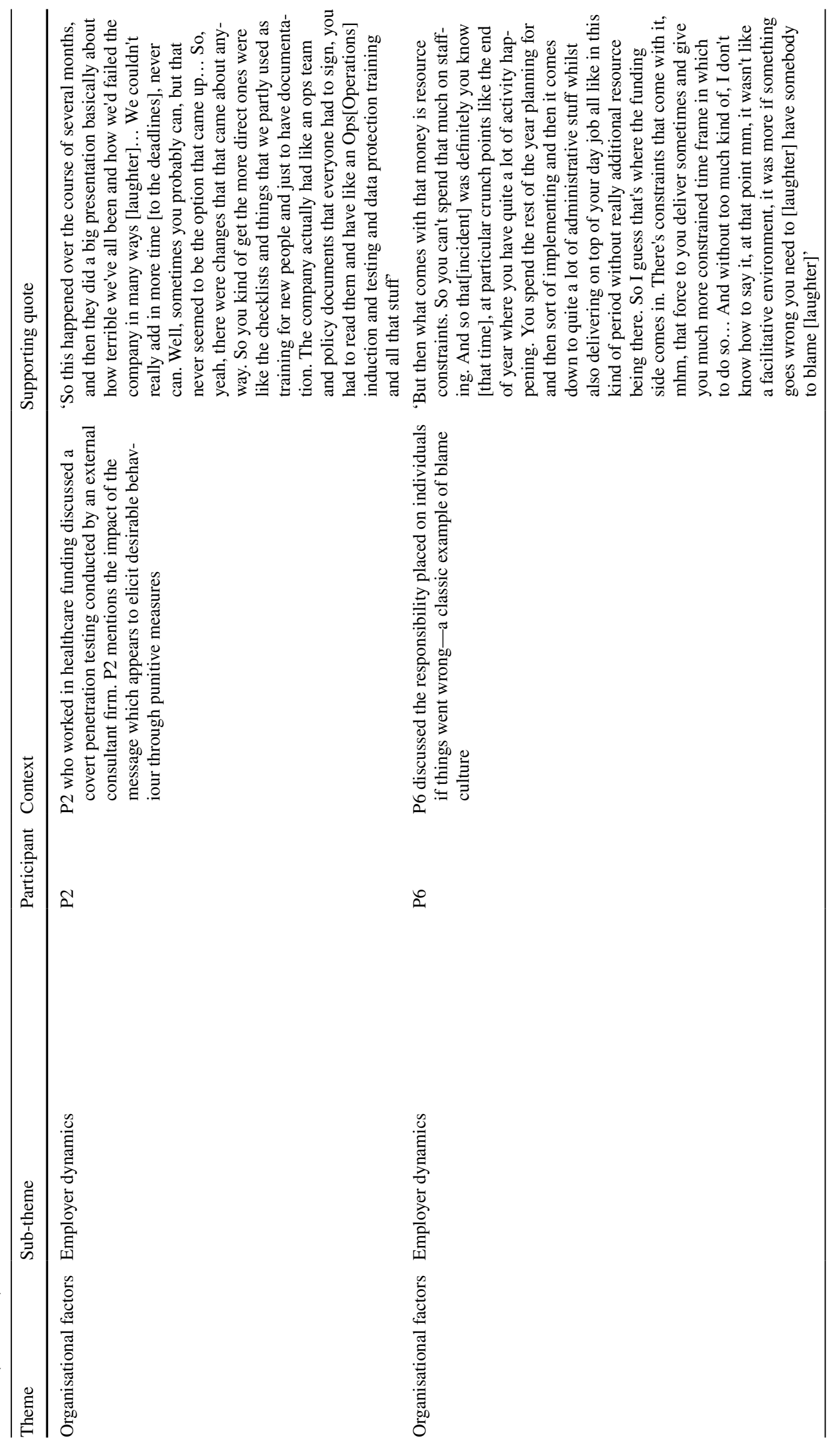




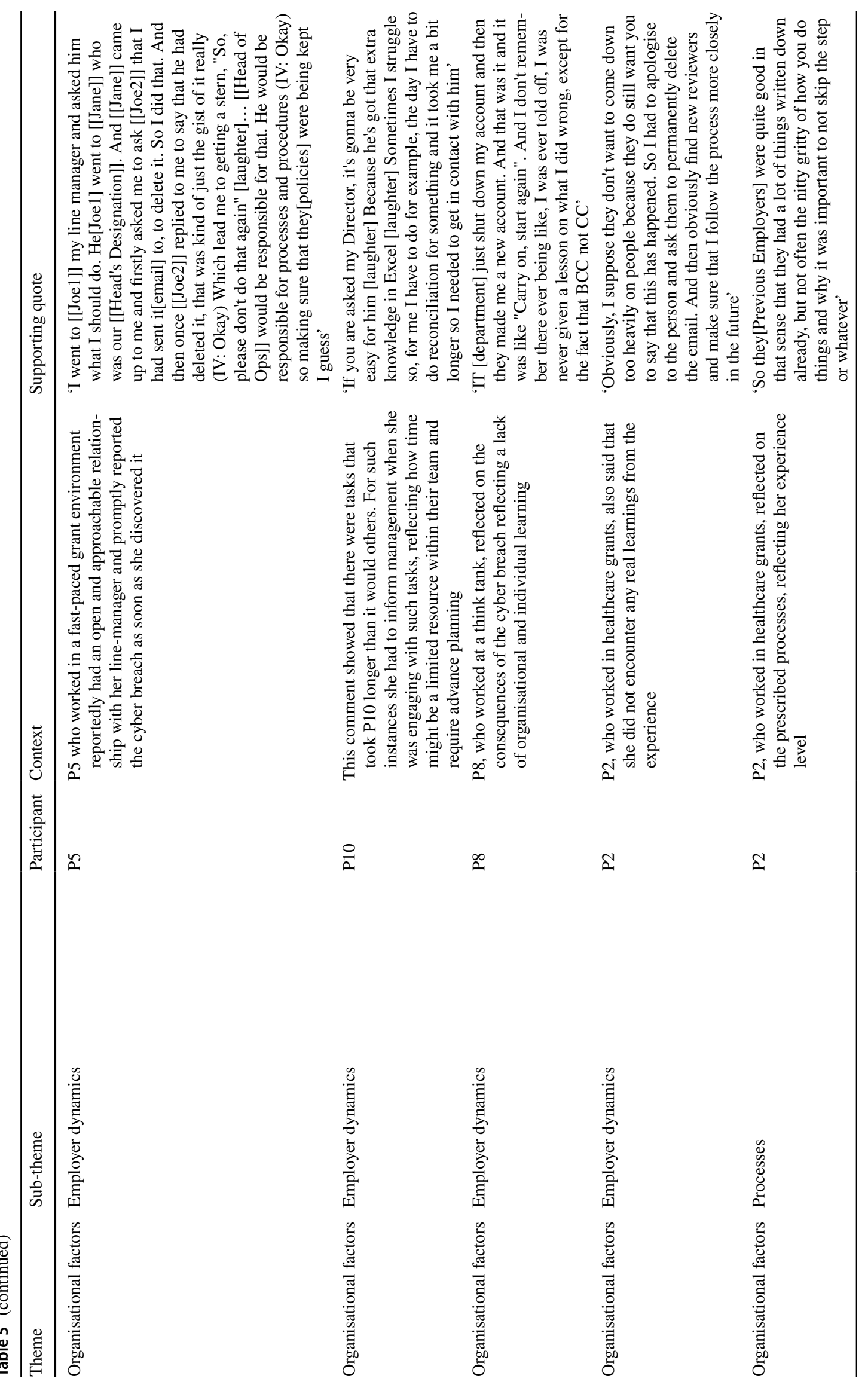




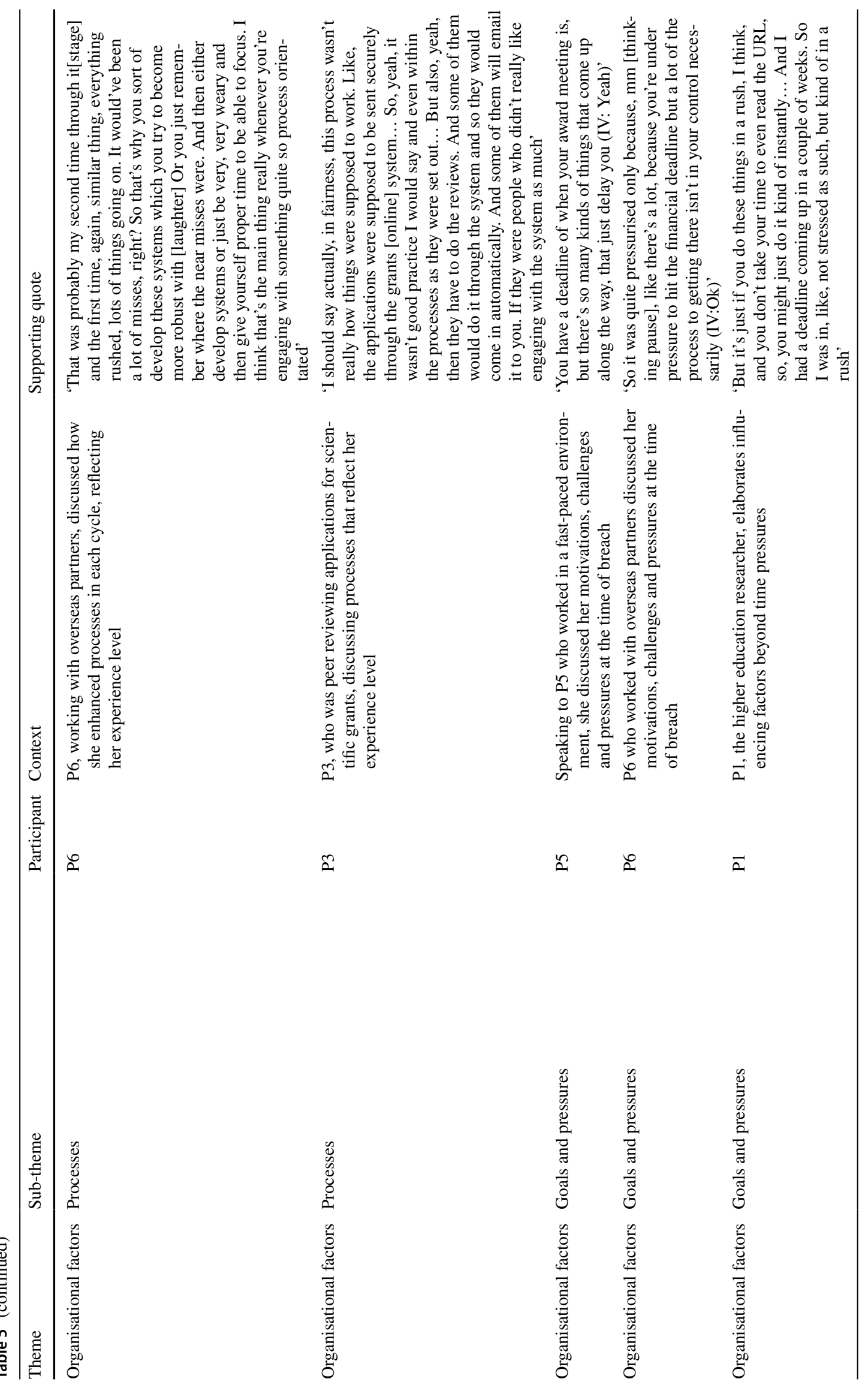




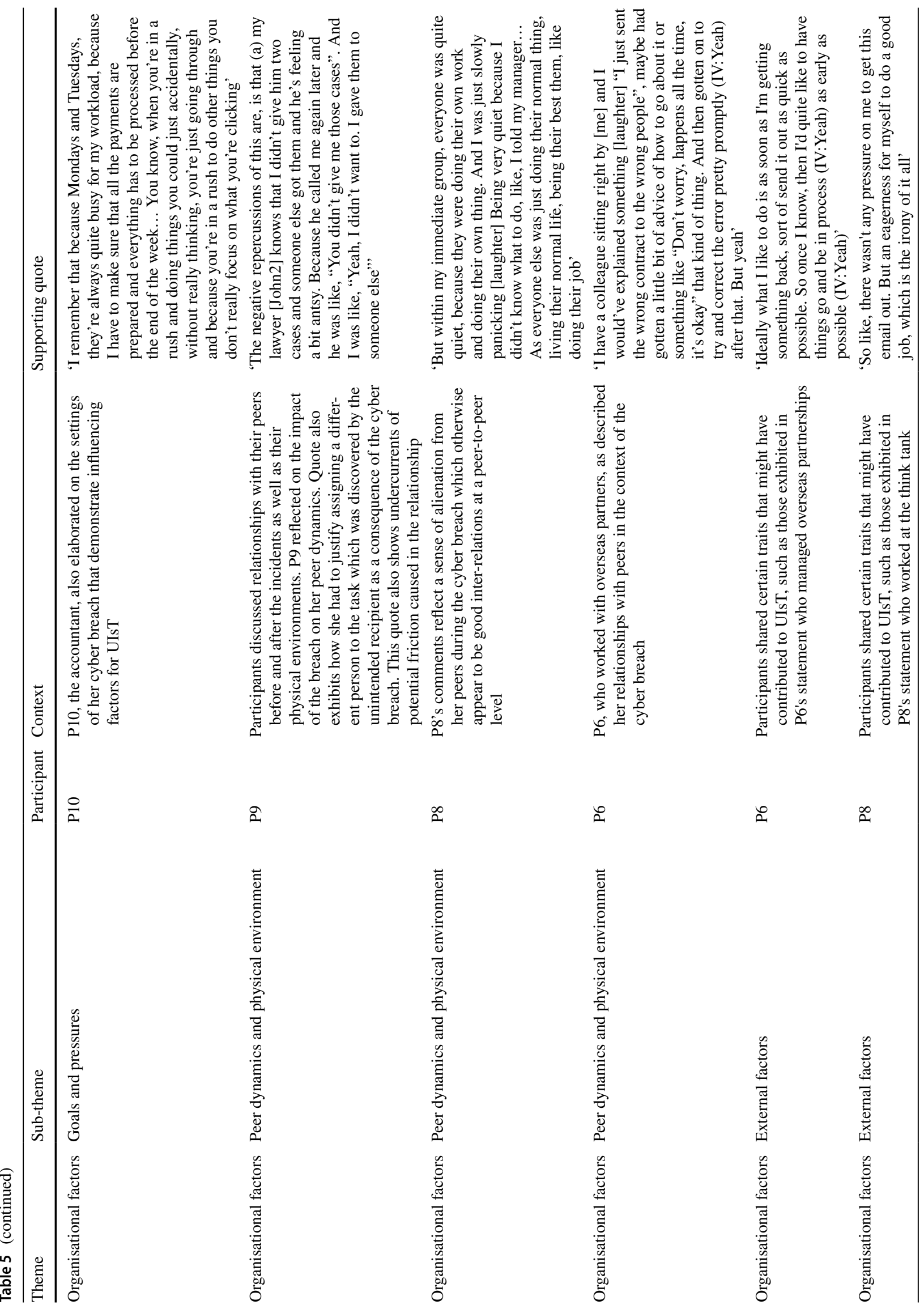




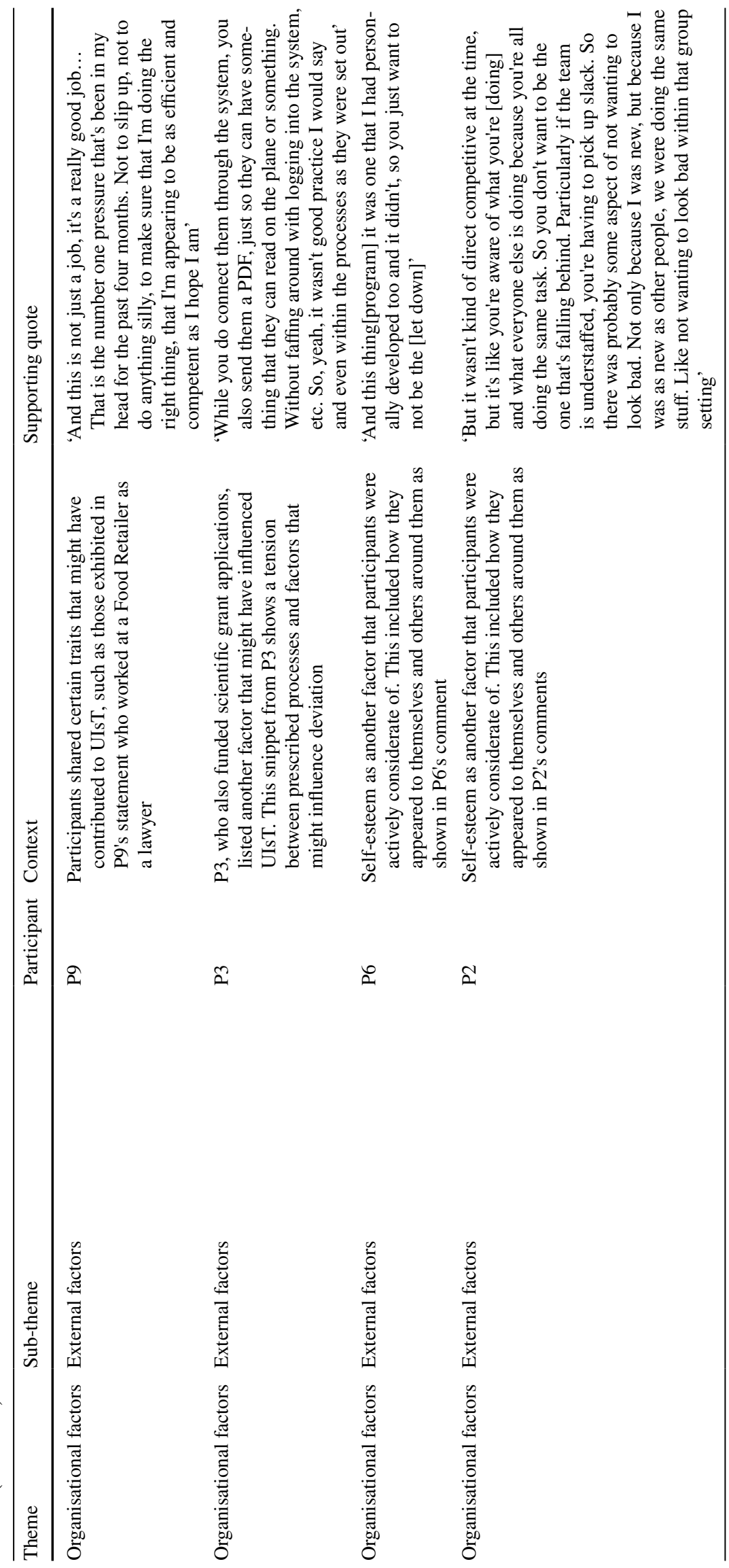


mentioned deviation in language and errors in the main body text to identify malicious content. Apart from the techniques used to evaluate technical elements of emails, participants also shared techniques that assisted them in making snap judgements about whether something was malicious. Techniques included reference to an incident that didn't occur or if something was too good to be true which reflected a mixture of Type 1 (intuitive thinking which is rapid and autonomous) and Type 2 (reflective thinking which requires working memory and other resources) thinking processes (Evans 2012) at different stages in their interactions. Having specific context to a conversation, the nature of the request from the sender and relying on a strategy similar to two-factor authentication (2FA) from establishments such as their banks also assisted them in their DM. While relying on 2FA approach to validate false alarms may appear to be a relaxed state, P4's technique reflects that her lived experience where no action was taken has resulted in things continuing safely. Interestingly, this strategy might aid in counteracting malicious emails that use urgency or time pressure techniques to lure targets.

\subsubsection{Acquired knowledge}

Participants largely appeared to have more confidence in their abilities compared to older generations specifically based on how various platforms were utilised whilst acknowledging that this confidence could be misplaced. Participants also considered informally acquired knowledge as more advantageous when safeguarding oneself against malicious content. Based on their personal and professional experiences all participants believed that they were comfortable with using technologies due to their exposure of growing up with it. This familiarity with technologies also brought a heightened risk awareness amongst all our participants for susceptibility to being scammed themselves and difficulty in identifying sophisticated scams. However, this heightened risk awareness might have stemmed from all our participants experiencing a cyber breach and not necessarily an attribute of comfort with technologies in general. Knowledge of cybersecurity was largely acquired through personal experiences, proactive online researching and social networks (online and offline). Some participants also mentioned other avenues such as classroom/lab based teachings and/or posters, marketing leaflets and bank app notifications that contributed to their knowledge but noted that these were less effective.

While lived experience and acquired knowledge help guide DM in our daily lives, the techniques deployed by individuals can subtly contribute towards indicators for assessing UIsT risk levels. If individuals are aware of latest techniques used by attackers, are confident with the use of technologies deployed to perform daily tasks or have internalised techniques to help identify malicious attempts it can provide a strengthening of defences (which we will discuss later in the framework). Equally, if individuals exhibit over-confidence or low levels of malicious content identification techniques (such as those discussed above) it might indicate a weakness in defences as end users would not possess the skillsets needed to make critical decisions in daily tasks that can result in UIsT being realised.

\subsection{Task factors}

Broad themes that directly linked to task factors in the context of the incident of a cyber breach included complexity of the task, speed (of the incident, discovery and response) and actions (to minimize impact, conclusion assumption and subsequent actions).

\subsubsection{Complexity of task}

Almost all participants reported tasks as being complex with many preceding or simultaneous actions that needed to be performed in order to successfully complete the task at hand. All our participants described using a mixture of techniques to deliver their respective complex tasks that included using templates, manually maintaining progress spreadsheets, pivot tables, e-mail merges, performing manual checks for accuracy, searching the internet, collating information, bespoke software platforms and various mass market software.

Beyond the use of technologies, participants frequently mentioned the human element that informed and influenced their task delivery. The human element was significant enough for participants to mould the process in a way that made the human related aspects in the task run smoothly. Since work is not conducted in a vacuum or in a linear way, all the tasks involved other people contributing in some way but almost all of the tasks involved other people actively feeding-in (internal and/or external) for the successful completion of the task. This collaboration with people appeared to influence participants' actions for how the task was conducted.

The active feeding-in from other people for the delivery of the task also created new information that needed to be processed and managed by the operators before future steps were selected, adding another layer to the complexity of the task. Participants interacted with information in numerous ways such as knowledge building, dissipating information to other people/systems and/or collecting information to inform their next step in the process.

Interacting with elements discussed above such as technologies, people and information contributes to the classification of complex tasks. In our study the delivering 
of such complex tasks facilitated new ways of working or in cases where processes were prescribed they were deemed insufficient in light of new information. We thus concluded that the complexity of a task can contribute to UIsT and processes should routinely be evaluated across all designations as indications of potential UIsT which we discuss in our framework later on.

\subsubsection{Speed of the incident, discovery and response}

While participants were engaged in delivering various complex tasks, unsurprisingly they all reported a very small window of time (seconds, minutes) over which the cyber breach occurred. The speed of the incident itself was so fast that some participants did not initially recognise they had experienced a cyber breach at all. Participants' experiences reflected how users at any given time are a-click-away from a cyber breach occurring which might be a feeling that was heightened in our participants post experiencing a cyber breach.

Not identifying if a breach had occurred straight away was common amongst our participants whilst the time taken to discover a breach varied (some cases it was within a minute, some took up to a week). The discovery of a breach was reliant on either on some form of checking process or delayed feedback. Examples included the use of checklists, other people identifying an anomaly with participant's account or a pop-up from the malicious software itself. In many cases this feedback was delayed and serendipitous suggesting an absence of clearly identified feedback loops and the possibility that some breaches may remain undetected for an indeterminate period of time if they are detected at all.

Once the cyber breach was discovered participants described a quick response speed to protect their cyber defences. This time window varied between a few minutes to a couple of days but in all cases was longer than the time window for the incident to occur depicting a typical gulf of evaluation (Norman 1986) for unintentional cyber breaches.

As the incident was so rapid in its nature and participants were performing complex tasks, we discuss how this element contributes to its fluctuating nature of UIsT which must be evaluated. We also discuss how assessing tasks to evaluate adequate feedback loops is essential and processes should be in place where individuals are familiarised with protocols in the event of a cyber breach to respond to threats promptly and effectively. Later on, in the discussion we introduce stop, think, ask, action, consequence or STAAC as a heuristic means of introducing reflection on actions taken.

\subsubsection{Actions}

Participants shared the actions they took prior to and during the breach. Contextual features appeared to assist participants in identifying safe content (such as an email from a known safe sender). However, applying the same principles or the misapplication of the same contextual cues made participants vulnerable to UIsT (i.e. receiving a virus from a known safe sender as their account had been compromised). This misapplication of contextual cues was often supported by assumptions being made to validate anomalies in interactions with people and systems in turn increasing the susceptibility to UIsT as users progressed in their DM to engage with malicious content.

Our results showed that during or immediately prior to experiencing a cyber breach, participants formed assumptions or had contextual cues that encouraged them to continue progressing with the task or underestimate the impact of a potential cyber breach. In all cases participants carried on with their normal duties until a cyber breach had actually been discovered. In contrast to not knowing if a cyber breach had occurred, once participants discovered that a cyber breach had taken place, they all performed some form of action to reduce the impact of the cyber breach. These actions included turning off equipment, reporting the incident to managers, contacting IT specialists and recalling the message.

Thus, actions can become critical for how to avoid or tackle a UIsT should it be realised. Findings from this heading feed into our framework by assessing individuals' understanding of outcomes from a cyber breach and ties in with a culture of empowerment which we discuss in Sect. 5.

\subsection{Accidents}

The broad themes that directly linked to the incident of cyber breach itself included training, expertise level, participants' trust in technology and errors (expecting errors from others, error in expectations and accepting errors in themselves).

\subsubsection{Training}

Participants were also asked if training could have helped them bypass the cyber breach. In our sample training was not seen to have much influence in deterring UIsT. While participants believed that training was slightly useful for general and theoretical awareness, they did not feel it could be good enough to bypass the cyber breach especially given the unintentional nature. Reinforcing earlier findings, participants identified informally acquired experience as more potent with all participants identifying sharing of knowledge with peers as valuable. Peer-to-peer sharing of knowledge 
and experiences through any medium (face-to-face, emails, forums, trainings) was believed to be a more effective form of learning and awareness than training for avoiding UIsT. The experience of a cyber breach appeared to have the most influence on avoiding future UIsT amongst participants. Our results indicate a direct correlation between UIsT and personalised experience, where first-hand experience can be the biggest deterrent, followed by experiences of people that are known to you in real life and training being deemed the least effective. We incorporate this finding in our framework and discuss this in greater detail in our discussion below to recommend personalised trainings that are audited and instilling a culture of empowerment to help mitigate UIsT.

\subsubsection{Expertise level, trust in technology and errors}

All participants identified themselves as having mid or advanced level of expertise at their jobs, listed in Table 6 . Eight participants shared that at the time of experiencing the cyber incident they were not reminded of similar lived experiences in the past, which are known as analogues to help aid decision making. Analogues were absent in instances where context was present for the participants (for instance being able to identify a popular ransomware's pop-up but not having similar lived experiences). Similarly, some participants shared that they had context as an analogue which meant that their lived experiences either encouraged them to proceed in the task despite reservations or identify the threat as it was unfolding (early detection). Analogues also included recalling previous cyber incidents, but none had escalated to a cyber breach. Overall, the experience of this incident was novel to a majority of the participants. In fact, for two of our participants analogues were present and it aided them in identifying subsequent steps to take moving forward and/ or anticipate consequences.

When discussing the cyber breach and exploring trust in technologies, participants had trust in technologies to protect users from harm such as malicious content blocked by firewalls. Participants also largely described a trusting relationship with technologies for automated elements within tasks. This included not cross-checking automated actions such as recipients that are auto populated for emails or not suspecting emails from within the organisation. In fact, this very trusting nature for systems to be secure resulted in a majority of the participants being victims to a cyber breach.

However, participants reported a distrust in technologies to perform a task correctly which included concerns such as manually cleaning data when exported to make it readable, reliability issues with exports, user problems such as early time-outs and forgetting passwords, all of which led to human input to overcome software limitations. Human input also led to participants making assumptions during various points of the cyber breach. For instance, actions that triggered the cyber breach were seen as insignificant even as the incident unfolded and its overall significance underestimated.

Assumptions were also made about various elements of the cyberthreat by some participants. This meant that interactions that seemed out of the ordinary were normalised by the participants as they could associate a reason for why the interaction was occurring. Lack of a feedback loop allowed participants to assume there was an error (for instance a broken link, wrongly attached file or an error in the process or human error) but only after they had been compromised.

Expertise levels can result in greater levels of analogues being present when deciding how to react to potential or unfolding UIsT. Analogues can assist in effective steps taken to contain the threat and are incorporated in our framework under evaluating automated tasks, assessing technical skill levels and evaluating effectiveness of guidelines in the event of a cyber breach. Individuals' trust in technologies is also a notable factor contributing to UIsT and this is addressed in our framework through evaluating software limitations and evaluating levels of trust in technologies amongst employees to strengthen defences. We incorporate errors in our framework by evaluating individuals' ability to question, share and challenge abnormal interactions that would indicate a culture
Table 6 Participant settings, levels of expertise and presence of analogues

\begin{tabular}{|c|c|c|c|c|c|}
\hline Participant & Cyber breach setting & Novice & Mid-level & Advanced & Analogue? \\
\hline $\mathrm{P} 1$ & Professional & & $\mathrm{x}$ & & Absent but context present \\
\hline $\mathrm{P} 2$ & Professional & & $\mathrm{x}$ & & Absent \\
\hline P3 & Professional & & & $\mathrm{x}$ & Present \\
\hline P4 & Professional & & $\mathrm{x}$ & & Absent \\
\hline P5 & Professional & & & $\mathrm{x}$ & Absent \\
\hline P6 & Professional & & & $\mathrm{x}$ & Absent \\
\hline P7 & Personal & & $\mathrm{x}$ & & Absent but context present \\
\hline P8 & Professional & & $\mathrm{x}$ & & Absent \\
\hline P9 & Professional & & & $\mathrm{x}$ & Present \\
\hline $\mathrm{P} 10$ & Professional & & $\mathrm{x}$ & & Absent \\
\hline
\end{tabular}


of knowledge sharing and empowerment. We discuss these elements in greater detail as part of our framework below.

\subsection{Organisational factors}

Another major theme that emerged from the data involved factors that related to the wider context under which participants performed their tasks, relating to organisational factors. Sub-categories included individual emotional responses, employer dynamics, processes, goals, pressures, peer dynamics, physical environment and external factors all of which appeared to interplay with the conditions that facilitated an unintentional cyber breach.

\subsubsection{Individual emotional responses}

A range of emotional responses were shared by participants during the interview following the cyber breach. Overall, there was a feeling of disbelief that the incident happened to them which elicited feelings of embarrassment and gullibility making them more cautious going forward. Participants shared having felt guilt and a sense of personal responsibility for being compromised. Furthermore, participants also shared feelings of frustration at themselves, software and processes that facilitated the cyber breach. As participants developed a level of caution post the cyber breach, we incorporate measuring levels of caution amongst employees to assess user vulnerabilities for UIsT, which we discuss later on.

\subsubsection{Employer dynamics}

Discussions at the interview also included employer's response to the cyber breach which was interesting as it shed light on some of the organisational factors that might have contributed to the UIsT incident that participants experienced.

Organisations' actions following a cyber breach appeared to fall short of strengthening cyber defences against UIsT. For instances in our findings, one example is a disclaimer ribbon on emails by the employer for employees to only interact with content that they recognise as safe. While this prompt can be a useful reminder it would not have prevented the UIsT experienced by P10 who recognised and trusted the sender. Beyond this trust, it would be problematic to know if the content was safe without exploring it as the malicious link did not have any identifiable anomalies to P10 i.e. it did not appear malicious. These types of notices can create a safety climate (Neal and Griffin 2004) as opposed to a safety culture (Reason 1998) and be seen to place full responsibility on individuals for their actions in-turn propagating a blame culture. Overall for our participants, where applicable, IT department personnel helped to combat the threat without placing blame but there were undertones of how the incident created more work for them. IT's countermeasures caused participants to experience downtime which disrupted their work with additional follow up tasks such as password resetting.

Participants also discussed their relationships with their line managers as well as any senior designations that were involved once a cyber breach had been identified. P2 mentions the impact of the employer's message which appears to elicit desirable behaviour through punitive measures. While measures such as checklists and improved processes can be a step in the right direction, in P2's case it is implemented in a way that placed responsibility on individuals if things went wrong-a classic example of blame culture where humans are seen as the weakest link in systems. This blame culture within organisations was also echoed in other participants' accounts.

A majority of the participants shared how they were able to work autonomously with approachable managers. This open communication correlated with participants' willingness to share the cyber breach with their managers early in the lifecycle of the threat. Participants also expressed having good immediate relationships with their peers and managers. However, participants' experiences beyond these immediate relationships largely reflected a blame culture discussed above. Additional tasks that were introduced by employers as a result of the cyber breach to safeguard against UIsT would be fundamentally inadequate, such as signing additional contractual documents or being told not to do that again. All our participants shared the sentiment of limited resources to perform their tasks (such as time and people) in the organisation which was believed to be a contributing factor to the cyber breach.

Overall, in all our participants there appeared to be a lack of organisational and individual learning and accountability from the cyber breach. We incorporated this finding in the framework by evaluating the effectiveness of prescribed processes. Findings from Employer Dynamics also contribute towards evaluating the effectiveness of guidelines in the event of the cyber breach, assessing individual's understandings of protocols in the event of a cyber breach, evaluating relationships between individuals and their managers, assessing stigma associated to incidents, levels of organisational communications about cyber incidents and assessing resources available to deliver tasks, all of which are discussed in greater detail in Sect. 5.

\subsubsection{Processes}

Participants described vague processes in place that generally guided them in how to perform various tasks. All our participants were relatively experienced in performing the tasks at hand and discussed how this familiarity allowed 
them to skip steps in the process that they did not deem important. Skipping steps or using unofficial channels was linked to saving time, efficiency or convenience, indicating established routine violations (Reason et al. 1990). Participants also discussed how processes had limitations, how their existing context facilitated their error but more importantly how they were aware of processes having limitations or potential for errors if followed as prescribed. These findings contributed towards the input of evaluating effectiveness of prescribed processes amongst skilled staff in our framework.

\subsubsection{Goals and pressures}

Discussing participants' goals at the time was important as it reflected their motivations for the task and how they performed it which might have contributed to the UIsT they experienced. When asked to declare time pressures experienced on a scale of 1-5 participants reported feeling under time pressure to deliver the task with an average score of 2.8 points/participant. Pressures did not solely emerge from time constraints (time pressure listed as 2.8 on a five-point scale) but also from deadlines and feeling a lack of control. Furthermore, participants also added other goals that motivated them which included: wanting to move on to another task, following the prescribed process, desire for a lower workload after successfully completing the task and being able to achieve a larger more important goal through the completion of the task at hand.

Stemming from this discussion about pressures participants went on to elaborate factors that were at play at the time of the incident. Participants elaborated on other factors beyond time pressures and experiencing factors such as planned deadlines and anticipated workload led to participants wanting to move on and rushing which in some cases was supported through implementing automatic behaviour to progress through the task. Findings from this theme contributed to the input of assessing individuals' motivations when delivering tasks to assist in identifying potential UIsT.

\subsubsection{Peer dynamics and physical environment}

When understanding the context in which cyber breaches occurred participants discussed relationships with their peers before and after the incidents as well as their physical environments. Overall, all our participants generally described having a friendly relationship with their peers which included being able to openly communicate with one another for advice and provide support through the cyber breach. They also described having a competitive relationship with their peers and everybody working autonomously to deliver their individual key performance indicators (KPIs). This led to participants feeling alienated from their peers and largely responsible to control the impact of the cyber incident as is shown in the examples corresponding to this heading in Table 5 .

Participants also described normal office environments with open plan spaces and normal noise levels. They all described physical environments where they could concentrate on tasks. In our results physical environment did not seem to be a contributor to UIsT but it did not also mitigate the threat from occurring. These findings contributed to evaluating relationships between peers and monitoring attention to detail in virtual tasks and physical environment within our framework. Having strong relationships at a peer level showed to have positively influenced mitigating against UIsT.

\subsubsection{External factors}

When speaking about their personal methods of working participants shared certain traits that might have contributed to UIsT. The interviews uncovered specific individual traits within our participants that might have been stimulated through various external factors. The traits reflected by our participants included being willing to take on and expect ad hoc work, being responsible for multiple projects, anticipating workload, taking personal responsibility for the delivery of tasks assigned to them and being detail oriented. Participants also appeared to possess good communication skills and the ability to ask for help which allowed participants to reduce the impact of the cyber breach. These traits also reflect a deeper connection to external factors such as job security and losing income for the organisation. Whist there appeared to be a tension between prescribed processes and factors that might influence deviation, all our participants also showed an active commitment to best practices and compassion for others who inputted into their tasks. In our study participants appeared to compromise on prescribed processes in favour of compassion for others. Beyond compassion for others, our discussions with all participants showed self-esteem as another factor that participants were actively considerate of. This included how they appeared to themselves and others around them.

The above findings pertaining to external factors appeared to influence the conditions that facilitated an unintentional cyber breach. These findings contributed to inputs for assessing prioritization of processes, commitment to best practices, personal responsibility taken by individuals when delivering tasks and levels of stigma associated to near-misses or accidents within our framework. We will now discuss these in greater detail in the following section. 


\section{Discussion}

The findings from our study discussed in the results section above were characterised as either features or action suggestions. Examples of features included data codes such as suspect logos, deviation in language, speed, compassion for others while action suggestions encompassed actions, such as turning equipment off, performing tasks and subsequent task elements, rushing, being reprimanded amongst other codes. One way of summarising our results in an accessible form is to adopt the convention of the Epidemiological Triangle most commonly used in public health communication. Classically, the triangle (or triad) represents the interplay between the Host (the putative victim of an infection), the Agent (the disease itself) and Environment in which both exist. The Host (the User) and Agent (the Exploit) may have various forms of intrinsic resistance and virulence, respectively, which are strengthened or weakened relative to each other by the environment (the Work Context), shown in in Fig. 3. This represents three vectors that may be militated against to reduce the chance of an incident (e.g., by prophylactic measures that strengthen the host, anti-disease steps that weaken the agents or by modifying the environment). This approach has also been used in the context of safety science (e.g., Gordon 1949; Haddon 1968). Here, we position the probability of a breach in relation to the features of the Exploit itself, the qualities of the User and their prior experience and the Work Context in which the breach occurs.

The overview exhibited through the Epidemiological Triangle allowed us to gain a deeper understanding of who would need to take responsibility for different elements of intervention, providing a structure to delivering any interventions. Accordingly, data codes were re-classified to create a framework (shown in Table 7) which informs the following discussion. For the purposes of this work a framework is a set of recommendations applicable in specific scenarios to reduce negative impact. Our framework proposes a five-pillar action plan listed as Outputs that can be achieved through 35 distinct Inputs. Based on our findings Input elements can be captured to assess the potential level of risk in a setting and therefore provide appropriate timely interventions. We believe this framework can be implemented by organisations that are interested in starting an UIsT program or as an evaluation tool for organisations that currently have one. We advise that this framework is conducted bi-annually or when organisational changes occur. It is also worth noting that we recommend tailored trainings that goes beyond traditional face-to-face teaching and are audited for their effectiveness.

\subsection{Technical defences}

Passive cyber defences are undoubtedly a good measure to serve as the first line of defence to protect networks against attacks. This should include virtual and physical spaces encompassed in examples discussed in the background section earlier. While our framework lists a few technical defence elements, such as conducting penetration testing and mapping all staff's ICT skillsets, it is recommended that all passive defences within technical defences are fully inclusive in the implementation of this framework. These include best practices for software architecture, monitoring user activities and devices, configuration, encryption, managing access points (including privileges), data management, updating software and regular audits. Passive defences are in-line with NCSC's and CERT's recommendations but in contrast to NCSC and CERT we do not recommend
Fig. 3 Summarising the results through an Epidemiological Triangle to represent the interplay between the three vectors of user, exploit and work context for UIsT

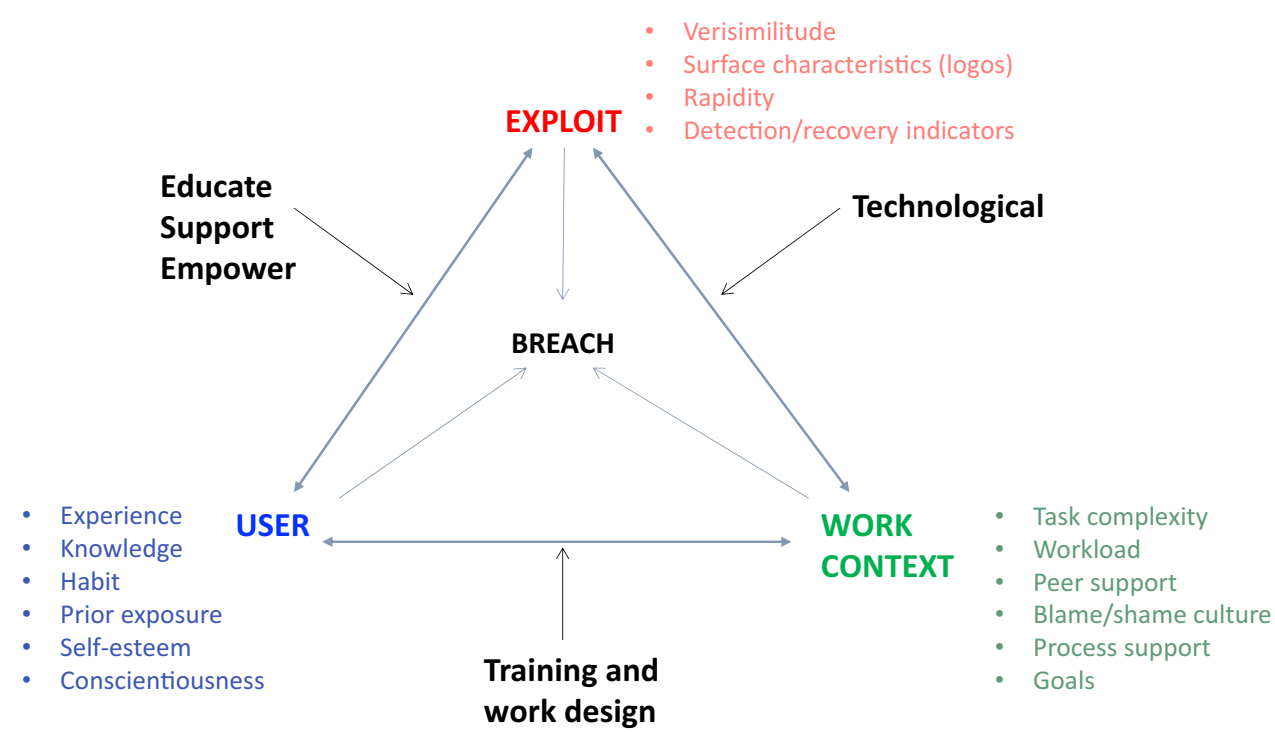


Table 7 Framework: each of the five outputs represent a pillar within the framework. Pillars encompass the elements that can be examined to indicate UIsT levels within an organisation and are informed by respective Inputs. These Inputs are variables that influence UIsT emerging from research study's findings and involve various socio- technical elements. This framework serves as a blueprint for identifying, intervening and mitigating UIsT and will inform future works that involve the representation and design of a tool that can be utilised by organisations

\begin{tabular}{lc}
\hline Outputs & Pillar no. \\
\hline User vulnerabilities to UIsT and recommendations to strengthen defences & 1 \\
The effectiveness of processes and facilitating a continuous improvement culture & 2 \\
Workload and sufficient resource allocation & 3 \\
Knowledge sharing and empowerment culture & 4 \\
Fluctuating vulnerabilities & 5 \\
\hline
\end{tabular}

Inputs Contributes to

Assess how comfortable individuals are with various technologies and platforms

Pillar 1

Assess how vulnerable users feel in their daily online interactions

Pillar 1

Assess physical working environments

Pillar 1

Assess individuals' ability to identify spear phishing scams to note vulnerabilities

Pillar 1

Assess individuals' existing experiences with malware or threats (including physical spaces)

Pillar 1

Assess individuals' knowledge base to evaluate understanding of current techniques used by hackers

Pillar 1

Assess individuals' susceptibility to rationalise abnormal behaviour or interactions

Pillar 1

Assess individuals' susceptibility to spear phishing

Pillar 1

Assess individuals' trust in technologies

Pillar 1

Assess the levels of how much individuals rely on their social networks (offline and online) to inform their decisions if faced

Pillar 1

with threats

Assess individuals' awareness of mainstream marketing campaigns against popular attacks

Pillar 1

Assess levels of retention from basic ICT teachings to establish levels of awareness

Pillar 1

Assess and map different skill levels between individuals in a diverse workforce

Assess individual's level of caution when interacting with suspicious or odd behaviour (online and physical parameters)

Pillar 1

Evaluate all tasks to identify missing feedback loops that indicate task completion

Pillar 1

Evaluate the effectiveness of prescribed processes amongst skilled/experienced staff

Pillar 1

Assess individuals' prioritization of processes

Pillar 2

Evaluate the effectiveness of prescribed processes amongst all designations

Pillar 2

Evaluate in-use software's limitations in prescribed processes

Pillar 2

Assess individuals' commitment to best practices set out by the company

Pillar 2

Evaluate processes for collaborative tasks that are automated

Pillar 2

Assess individuals' technical skill levels

Pillar 2

Assess individuals' levels of personal responsibility felt when delivering tasks assigned to them

Pillar 2

Assess resources available to individuals to deliver tasks

Pillar 3

Assess individuals' motivations when delivering tasks

Pillar 3

Assess individuals' ability and willingness to take on additional tasks

Pillar 3

Assess levels of stigma associated with experiences of near misses and accidents that result in cyber incidents and

Pillar 3

cyber breaches across all levels

Assess levels of communication about cyber incidents

Pillar 4

Assess individuals' understanding of outcomes that result from accidents

Pillar 4

Evaluate effectiveness of current guidelines in the event of a cyber breach

Pillar 4

Pillar 4

Evaluate individuals' understanding of protocols in the event of a cyber breach

Pillar 4

Evaluate individuals' ability to question, share and challenge abnormal interactions

Pillar 4

Evaluate relationships between individuals and managers across all levels

Pillar 4

Evaluate relationships between peers across all levels

Pillar 4

Assess individuals' level of attention to detail (online and physical parameter)

Pillar 5 
restriction of devices or features as it can encourage users to create unauthorised or unmonitored back channels for delivering tasks. Instead in the following section we propose evaluating and noting how tasks are conducted so suitable defences can be implemented. While it would be desirable to develop in-house technical skills through formal training as suggested by NCSC, as part of the first pillar we recommend mapping existing skills to identify talent that can be utilised and developed across all designations. We also do not recommend the use of active cyber defences such as those used in SOFIT. Active defences can create complexities for implementation as they are dependent on permissibility in local laws (such as packet sniffing) as they infringe on individual privacy and can foster distrust between the organisation and individuals. Active defences also do not appear to be effective for safeguarding against UIsT as active monitoring against unintentional actions is fundamentally inapplicable.

\subsection{Sociotechnical defences}

Points contributing to each outcome in our framework are not separated as belonging to individual, technological or organisational contexts but rather findings are integrated to provide effective solutions that can identify, intervene and mitigate UIsT. The framework lists inputs which provide objectives for evaluations and outputs which are the five pillars to help gauge levels of vulnerability to UIsT and provide recommendations to strengthen defences. The framework introduces 'Stop, Think, Ask, Action, Consequence' or STAAC that is used to foster Type 2 thinking that counteracts UIsT. STAAC can be used prior, during or after a threat has been realised.

\subsubsection{User vulnerabilities to UIsT and recommendations to strengthen defences}

The first pillar of our framework provides an assessment report to benchmark existing vulnerabilities to UIsT within an organisation. This is done by evaluating 15 distinct points listed as inputs in Table 7.

In contrast to CERT, SOFIT and other popular models that rely on psychological and behavioural profiling our framework does not assign methods or people for evaluating elements within inputs. As this framework specifically targets UIsT we also do not require any individuals (such as HR personnel or peers) deducing individual personality traits or reporting suspicious behaviours that are geared towards identifying intentional IsT. Instead to benchmark vulnerabilities we evaluate individual's comfortability with various technologies, risk awareness levels (in-line with NCSC), individual lived experience and acquired levels of knowledge to identify malicious content and individual susceptibility to rationalise anomalies in interactions. We also assess physical environment for environmental stressors that are indicated in SOFIT and individual's trust in technologies to safeguard against malicious content. As part of the actions to evaluate UIsT, it encompasses educating and raising awareness amongst individuals through traditional and hands-on training as suggested by NCSC and CERT.

\subsubsection{The effectiveness of processes and facilitating a continuous improvement culture}

Our results support a link between task processes and the risk of breaches and validates Liginlal et al.'s (2009) recommendation for developing effective processes to tackle UIsT. In addition to effective processes our findings highlighted the importance of individuals understanding why steps within a process are important. For cases where explicit processes existed, our participants who had good expertise at performing their tasks, skipped steps as the importance of following each step was not communicated and had resulted in near-misses than accidents in the past but never an actual breach.

In this section of the framework, we recommend the evaluation of processes with the help of staff who possess good expertise for performing their assigned tasks. In contrast to Liginlal et al. who focus on addressing the lack of expertise through training, we emphasize the importance of working with expert individuals to identify heuristics and shortcuts that can facilitate UIsT. It also allows creation of processes that reflect 'work as done' as opposed to 'work as imagined' (Hollnagel 2017, Suchman 1985). At this stage we also assess the processes' effectiveness, individual prioritisation techniques that might compromise processes, conducting task analysis to device effective and improved processes as the delivery of the task changes, evaluating tasks that include automated elements and mapping software limitations that foster undesirable practices being implemented. While it is important to carefully consider implementing new systems that can facilitate new errors (Liginlal et al. 2009), it is also critically important to evaluate existing systems' effectiveness and suitability for prescribed processes. As part of this stage, we also evaluate individuals' commitment to best practices, trade-offs made and mapping individual technical skill levels when delivering tasks as all these factors were shown to influence UIsT.

\subsubsection{Workload and sufficient resource allocation}

Assessment of workload and allocating sufficient resources is the third pillar of this framework. SOFIT (Greitzer et al. 2018) includes workload as an indicator and while time 
pressures are the cornerstones for CERT and Nurse et al.'s frameworks for indicating UIsT our results highlighted additional interlinked factors. These factors included individuals feeling personally responsible for delivering tasks and allocation of sufficient resources (which included time and people) which is discussed in greater detail in the previous section. Individuals' motivations for delivering tasks are also important to consider as motivations such as rushing or meeting unrealistic deadlines (in-line with SOFIT) can support human fallibility. In addition, our framework incorporates organisational expectation for individuals to undertake new tasks that were also seen to be linked to UIsT.

In contrast with SOFIT, our results did not suggest strong links between UIsT and poor team management as participants reportedly enjoyed good interpersonal relationships with their direct line managers and peers. We did not find evidence for the mismatch between expectations and abilities listed in Liginlal et al.'s framework as participants had mid to advanced level of expertise to perform assigned tasks. We also did not find any evidence to support ambiguous goal setting or poor communication of goals (no data gathered for poor morale) all of which are factors included in SOFIT. Therefore, these elements have not been included in the framework.

\subsubsection{Knowledge sharing and empowerment culture}

NCSC, CERT, Liginlal et al. and SOFIT all include blame culture as being an indicator of UIsT and recommend instilling a culture of empowerment to counteract IsT. Our results also found that alongside an empowerment culture, which influenced UIsT, how knowledge was availed and shared between individuals had an impact on UIsT risks.

This section of the framework evaluates the culture of an organisation through assessing stigma and levels of organisational communication associated to cyber incidents and breaches. It also evaluates individual's understanding of outcomes that are associated to cyber breaches, effectiveness of guidelines in the event of an attack and individual understanding of subsequent protocols, ability to challenge abnormal interactions and inter-organisational relationship dynamics. We recommend creating security protocols based on STAAC. As UIsT is rapid in its nature and participants noticed anomalies that they ignored we introduce STAAC to assist individuals in slowing down and thinking through their actions at various points in the cyber breach (prior or during) which can help identify, intervene and mitigate UIsT. As suggested by NCSC, our framework also promotes the importance of practicing cyber breach protocols through drills (incorporating STAAC) and establishing an incident reporting culture through creating platforms (off-line and online) for knowledge sharing.

\subsubsection{Fluctuating vulnerabilities}

As the final pillar and further to the relevant frameworks discussed above, we propose a regular assessment of fluctuating vulnerabilities discussed above that can influence UIsT. As UIsT is changing in its nature due to these fluctuating vulnerabilities, we suggest using several indicators, such as evaluating attention to detail (online and physical parameter), to formulate recommendations.

\section{Conclusions, limitations and future work}

As attacks get increasingly sophisticated, well-intentioned employees have become prime targets for hackers to enable successful cyberattacks such as ransomware. UIsT continues to constitute a major threat to organisational assets that can result in significant disruption to operations as well as substantial financial and reputational damages. In order to tackle this threat effectively organisations need to have a clear understanding of UIsT and factors that influence it in complex environments. In this paper we discussed UIsT as a separate and distinct phenomenon to intentional IsT to build a better understanding of this threat. We also proposed an amalgamated framework developed through grounded theory applied to interview datasets and further refined through the Epidemiological Triangle.

As a result of our findings the framework incorporated new findings and enhanced existing elements from relevant frameworks designed to tackle IsT (intentional and UIsT). Existing elements that are utilised in our framework include the use of passive defences (NCSC and CERT), mapping in-house technical skills across all designations to build talent (NCSC), risk awareness (NCSC), evaluating physical environmental stressors (SOFIT), educating and raising awareness through training (NCSC and CERT), evaluating processes (Liginlal et al.), monitoring time pressures (CERT and Nurse et al.) and instilling an organisational culture of empowerment (NCSC, CERT, Liginlal et al., SOFIT). New features introduced in this framework include the following:

- Listing of rationale behind steps within processes, designing and evaluating processes with staff who possess expertise at performing assigned tasks and periodically evaluating processes' effectiveness through 'work as conducted' are essential

- New interlinked factors to time pressures are incorporated

- Knowledge attainment and sharing in addition to an empowerment culture is recommended

- UIsT involves fluctuating vulnerabilities that must be known, monitored and addressed 
We envisage that the framework presented in this paper at Stage 1 could be adopted by small and large organisations alike to build and strengthen effective sociotechnical defences against cyber breaches stemming from UIsT. STAAC is introduced as a guiding principle to help individuals identify, intervene and mitigate against UIsT through Type 2 thinking which is embedded through regularly audited trainings, workshops and drills. Our Stage 1 framework sets out five pillars listed as outcomes which might be achieved through the evaluation of various Inputs that appear to influence UIsT.

Limitations of this work emerge from snowball sampling that might limit the diversity of participants and in turn the generalisability of the findings. Some participants were already known to the interviewer; this facilitated candid and honest discussions, but this rapport might have also influenced participants' responses to some degree. As participants were asked to recall an incident in the past, whilst this incident was significant in their lived experiences, the application of recall and memory bias might unintentionally include or exclude information that might be significant for the findings.

Future directions stemming from the research presented in this paper are currently being explored. This includes the adoption of the framework at an organisation(s) for validation. Adoption of the framework in industry settings can provide a measure of effectiveness of the suggestions through incident rates prior and post adoption. It can also provide an opportunity to collect feedback from end users about the design and display of outputs (UX). Future work might also involve the creation of recommendations (what steps to take) that might strengthen inputs if they are reportedly weak and in turn strengthen pillars and ultimately defences against UIsT whilst maintaining human agency and empowerment within the system.

Acknowledgements The first author is supported by the Horizon Centre for Doctoral Training at the University of Nottingham (UKRI Grant No. EP/L015463/1). The last author is supported by the Engineering and Physical Sciences Research Council: Horizon Digital Economy Research Hub (EPSRC Grant No. EP/G065802/1).

Open Access This article is licensed under a Creative Commons Attribution 4.0 International License, which permits use, sharing, adaptation, distribution and reproduction in any medium or format, as long as you give appropriate credit to the original author(s) and the source, provide a link to the Creative Commons licence, and indicate if changes were made. The images or other third party material in this article are included in the article's Creative Commons licence, unless indicated otherwise in a credit line to the material. If material is not included in the article's Creative Commons licence and your intended use is not permitted by statutory regulation or exceeds the permitted use, you will need to obtain permission directly from the copyright holder. To view a copy of this licence, visit http://creativecommons.org/licenses/by/4.0/.

\section{References}

Agrafiotis I, Nurse JCR, Buckley O, Legg P, Creese S, Goldsmith M (2015) Identifying attack patterns for insider threat detection. Comput Fraud Secur. https://doi.org/10.1016/S1361-3723(15) 30066-X

Ani U, Daniel N, Oladipo F, Adewumi S (2018) Securing industrial control system environments: the missing piece. J Cyber Secur Technol. https://doi.org/10.1080/23742917.2018.1554985

Bearman C, Bremner P (2013) A day in the life of a volunteer incident commander: errors, pressures and mitigating strategies. Appl Ergon 44(3):488-495. https://doi.org/10.1016/j.apergo.2012.10. 011

Bell A, Rogers M, Pearce J (2019) The insider threat: behavioral indicators and factors influencing likelihood of intervention. Int J Crit Infrastruct Prot. https://doi.org/10.1016/j.ijcip.2018.12.001

Bhaskar R (1989) Reclaiming reality: a critical introduction to contemporary philosophy. Sage, London

Bishop M, Engle S, Peisert S, Whalen S, Gates C (2008) We have met the enemy and he is us. In: Proceedings of the 2008 new security paradigms workshop. https://doi.org/10.1145/1595676.1595678

Canham M, Posey C, Bockelman P (2020) Confronting information security's elephant, the unintentional insider threat. Int Conf Hum Comput Interact HCI. https://doi.org/10.1007/978-3-030-504397_22

Cappelli D, Desai A, Moore A, Shimeall T, Weaver E, Willke B (2007) Management and education of the risk of insider threat (MERIT): mitigating the risk of sabotage to employers information, systems, or networks. Carnegie Mellon Univ. https://doi.org/10.1184/R1/ 6575231.v1

Cappelli D, Desai A, Moore A, Shimeall T, Weaver E, Willke B (2008) Management and education of the risk of insider threat (MERIT): system dynamics modeling of computer system. Carnegie Mellon University, Pittsburgh. https://resources.sei.cmu.edu/library/assetview.cfm?assetid $=52324$. Accessed 17 Sep 2020

CERT Insider Threat Team (2013) Unintentional insider threats: a foundational study. Softw Eng Inst. https://doi.org/10.1184/R1/ 6585575.v1

Chattopadhyay P, Wang L, Tan Y-P (2018) Scenario-based insider threat detection from cyber activities. IEEE Trans Comput Soc Syst. https://doi.org/10.1109/TCSS.2018.2857473

Dice Staff (2020) Cybersecurity in 2021: 5 Trends Security Pros Need to Know. Dice Insights. https://insights.dice.com/2020/12/14/ cybersecurity-in-2021-5-trends-security-pros-need-to-know/. Accessed 17 Dec 2020

Evans JSBT (2012) Spot the difference: distinguishing between two kinds of processing. Mind Soc 11:121-131. https://doi.org/10. 1007/s11299-012-0104-2

Glaser BG, Strauss AL (1967) The discovery of grounded theory: strategies for qualitative research. Routledge, New York

Goethals PL, Hunt ME (2019) A review of scientific research in defensive cyberspace operation tools and technologies. J Cyber Secur Technol. https://doi.org/10.1080/23742917.2019.1601889

Gordon J (1949) The epidemiology of accidents. Am J Public Health Nations Health 39:504-515

Greitzer FL, Hohimer RE (2011) Modeling human behavior to anticipate insider attacks. J Strateg Secur. https://doi.org/10.5038/19440472.4.2.2

Greitzer F, Purl J, Leong YM, Becker DES (2018) SOFIT: sociotechnical and organizational factors for insider threat. In: 2018 IEEE security and privacy workshops. https://doi.org/10.1109/SPW. 2018.00035

Haddon W (1968) The changing approach to the epidemiology, prevention, and amelioration of trauma: the transition to approaches etiologically rather than descriptively based. Am J Public Health 
Nations Health 58:1431-1438. https://doi.org/10.2105/ajph.58.8. 1431

Hadlington L (2018) The "human factor" in cybersecurity: exploring the accidental insider. In: McAlaney J, Frumkin LA, Benson V (eds) Psychological and behavioral examinations in cyber security. IGI Global, pp 46-63. https://doi.org/10.4018/978-1-5225-40533.ch003

Hoda R, Noble J, Marshall S (2010) Using grounded theory to study the human aspects of software engineering. HAoSE . https://doi. org/10.1145/1938595.1938605

Hoffman RR, Crandall B, Shadbolt N (1998) Use of the critical decision method to elicit expert knowledge: a case study in the methodology of cognitive task analysis. Hum Factors. https://doi.org/ $10.1518 / 001872098779480442$

Hollnagel E, Wears RL, Braithwaite J (2015) From Safety-I to SafetyII: a white paper. University of Southern Denmark, University of Florida and Macquarie University. https://doi.org/10.13140/ RG.2.1.4051.5282

Hollnagel E (2017) Why is work-as-imagined different from work-asdone? In: Wears RL, Hollnagel E (eds) Resilient health care, vol 2. CRC Press, Boca Raton

Hunker J, Probst C (2011) Insiders and insider threats—an overview of definitions and mitigation techniques. J Wirel Mob Netw Ubiquitous Comput Dependable Appl. https://doi.org/10.22667/JOWUA. 2011.03.31.004

Kammüller F, Probst CW (2013) Invalidating policies using structural information. In: 2013 IEEE security and privacy workshops. https://doi.org/10.1109/SPW.2013.36

Keeney M, Kowalski E, Cappelli D, Moore A, Shimeall T, Rogers S (2005) Insider threat study: computer system sabotage in critical infrastructure sectors. National Threat Assessment CTR, Washington. https://apps.dtic.mil/dtic/tr/fulltext/u2/a636653.pdf. Accessed 23 Sep 2020

Klein GA, Calderwood R, MacGregor D (1989) Critical decision method for eliciting knowledge. In: IEEE transactions on systems, man, and cybernetics. https://doi.org/10.1109/21.31053

Legg PA, Buckley O, Goldsmith M, Creese S (2017) Automated insider threat detection system using user and role-based profile assessment. IEEE Syst J. https://doi.org/10.1109/JSYST.2015.2438442

Liginlal D, Sim I, Khansa L (2009) How significant is human error as a cause of privacy breaches? An empirical study and a framework for error management. Comput Secur. https://doi.org/10.1016/j. cose.2008.11.003

Magklaras G, Furnell S (2002) Insider threat prediction tool: evaluating the probability of IT misuse. Comput Secur. https://doi.org/ 10.1016/S0167-4048(02)00109-8

Morel B (2011) Artificial intelligence and the future of cybersecurity. In: Proceedings of the 4th ACM workshop on security and artificial intelligence (AISec '11), Association for Computing Machinery, New York. https://doi.org/10.1145/2046684.2046699

Mundie DA, Perl S, Huth CL (2013) Toward an ontology for insider threat research: varieties of insider threat definitions. In: 2013 third workshop on socio-technical aspects in security and trust. https://doi.org/10.1109/STAST.2013.14

Muller MJ, Kogan S (2010) Grounded theory method in HCI and CSCW. IBM Center for Social Software, Cambridge, pp 1-46

NCSC (2012) 10 steps to cyber security: guidance on how organisations can protect themselves in cyberspace, including the 10 steps to cyber security. https://www.ncsc.gov.uk/collection/10-steps-tocyber-security. Accessed 27 Aug 2020

Neal A, Griffin MA (2004) Safety climate and safety at work. In: Barling J and Frone MR (eds) The psychology of workplace safety.
American Psychological Association, pp 15-34. https://doi.org/ 10.1037/10662-002

Norman DA (1986) Cognitive engineering, chapter 3. In: Norman DA, Draper SW (eds) User centered system design; new perspectives on human-computer interaction. Lawrence Erlbaum Associates, New Jersey

Nurse JRC, Buckley O, Legg PA, Goldsmith M, Creese S, Wright GRT, Whitty M (2014) Understanding insider threat: a framework for characterising attacks. In: 2014 IEEE security and privacy workshops. https://doi.org/10.1109/SPW.2014.38

Ogiela MR, Ogiela U (2012) Linguistic protocols for secure information management and sharing. Comput Math Appl. https://doi.org/ 10.1016/j.camwa.2011.10.038

Pauley K, Flin R, Yule S, Youngson G (2011) Surgeons' intraoperative decision making and risk management. Amjsurg 202(4):375-381. https://doi.org/10.1016/j.amjsurg.2010.11.009

Plant KL, Stanton NA (2013) What is on your mind? Using the perceptual cycle model and critical decision method to understand the decision-making process in the cockpit. Ergonomics 56(8):1232 1250. https://doi.org/10.1080/00140139.2013.809480

Predd J, Pfleeger SL, Hunker J, Bulford C (2008) Insiders behaving badly. IEEE Secur Priv. https://doi.org/10.1109/MSP.2008.87

Reason J (1998) Achieving a safe culture: theory and practice. Work Stress 12(3):293-306. https://doi.org/10.1080/026783798082568 68

Reason J, Manstead A, Stradling S, Baxter J, Campbell K (1990) Errors and violations on the roads: a real distinction? Ergonomics 33(10 11):1315-1332. https://doi.org/10.1080/00140139008925335

Schuh G, Potente T, Wesch-Potente C, Weber AR, Prote JP (2014) Collaboration mechanisms to increase productivity in the context of Industrie 4.0. Proc CIRP 19:51-56. https://doi.org/10.1016/j. procir.2014.05.016

Siegel H (2004) Relativism. In: Niiniluoto I, Sintonen M and J Wolenski (eds) Handbook of Epistemology. Springer, Dordrecht, pp $747-780$

Suchman L (1985) Plans and situated actions: the problem of humanmachine communication. Cambridge University Press, Cambridge

Vanderhaegen F, Wolff M, Mollard R (2020) Non-conscious errors in the control of dynamic events synchronized with heartbeats: a new challenge for human reliability study. Saf Sci. https://doi.org/10. 1016/j.ssci.2020.104814

Verizon (2020) Data Breach Investigations Report. https://enterprise. verizon.com/resources/reports/2020-data-breach-investigationsreport.pdf. Accessed 12 Jan 2021

Wong BLW (2004) Critical decision method data analysis. In: Diaper D, Stanton N (eds) The handbook of task analysis for humancomputer interaction. Lawrence Erlbaum Associates, New Jersey, pp 327-346

Woods DD, Hollnagel E (2006) Joint cognitive systems: patterns in cognitive systems engineering. CRC Press, Boca Raton

Zargar A, Nowroozi A, Jalili R (2016) XABA: a zero-knowledge anomaly-based behavioral analysis method to detect insider threats. In: 2016 13th International Iranian society of cryptology conference on information security and cryptology (ISCISC). https://doi.org/ 10.1109/ISCISC.2016.7736447

Publisher's Note Springer Nature remains neutral with regard to jurisdictional claims in published maps and institutional affiliations. 